JOURNAL OF THE

AMERICAN MATHEMATICAL SOCIETY

Volume 22, Number 4, October 2009, Pages 909-940

S 0894-0347(09)00640-7

Article electronically published on April 30, 2009

\title{
SCHUBERT CALCULUS AND REPRESENTATIONS OF THE GENERAL LINEAR GROUP
}

\author{
E. MUKHIN, V. TARASOV, AND A. VARCHENKO
}

\section{INTRODUCTION}

It has been known for a long time that the intersection index of the Schubert varieties in the Grassmannian of the $N$-dimensional planes coincides with the dimension of the space of invariant vectors in a suitable tensor product of finite-dimensional irreducible representations of the general linear group $\mathfrak{g l}_{N}$.

There is a natural commutative algebra acting on such a space of invariant vectors, which we call the Bethe algebra. The Bethe algebra is the central object of study in the quantum Gaudin model; see [G. In this paper, we construct an algebra isomorphism between the Bethe algebra and the scheme-theoretic intersection of the Schubert varieties. We also show that the representation of the Bethe algebra on the space of invariant vectors is naturally isomorphic to the coregular representation of the scheme-theoretic intersection of the Schubert varieties (in fact, the regular and coregular representations of that algebra are isomorphic). Thus we explain and generalize the above-mentioned coincidence of the numbers.

The existence of such a strong connection between seemingly unrelated subjects (Schubert calculus and integrable systems) turns out to be very advantageous for both.

On the side of Schubert calculus, using a weaker form of the relation, we succeeded in proving the B. and M. Shapiro conjecture in MTV2. An alternative proof is given in the present paper. Moreover, we are now able to deduce that the Schubert varieties corresponding to real data intersect transversally; see Corollary 6.3. The transversality of the intersection was a long-standing conjecture; see [EH], $[\mathbf{S}$.

The transversality property can be reformulated as the following statement: the number of monodromy free monic Fuchsian differential operators of order $N$ with $k+1$ singular points, all of them lying on a line or a circle, and prescribed exponents at the singular points equals the multiplicity of the trivial $\mathfrak{g l}_{N}$-module in the tensor product of $k+1$ irreducible $\mathfrak{g l}_{N}$-modules with highest weights determined by the exponents at the critical points.

On the side of the integrable systems, we obtain a lot of new information about the Bethe algebra; see Theorem 6.1. In particular, we are able to show that all eigenspaces of the Bethe algebra are one-dimensional for all values of the parameters. Since it is known that the Bethe algebra is diagonalizable for real data, our

Received by the editors January 11, 2008.

2000 Mathematics Subject Classification. Primary 17B67, 14P05, 82B23.

The first author is supported in part by NSF grant DMS-0601005.

The second author is supported in part by RFFI grant 05-01-00922.

The third author is supported in part by NSF grant DMS-0555327.

(C)2009 American Mathematical Society 909

Reverts to public domain 28 years from publication 
result implies that the spectrum of the Bethe algebra is simple in this case, and hence it is simple generically. We also show that the Bethe algebra is a maximal commutative subalgebra in the algebra of linear operators.

In addition, an immediate corollary of our result is a bijective correspondence between eigenvalues of the Bethe algebra and monic differential operators whose kernels consist of polynomials only; see Theorem 6.1. parts (v), (vi). The obtained correspondence between the spectrum of the Bethe algebra and the differential operators is in the spirit of the geometric Langlands correspondence; see $\mathrm{F}$.

We obtained similar results for the Lie algebra $\mathfrak{g l}_{2}$ and the Grassmannian of two-dimensional planes in MTV3. The crucial difference between MTV3 and the present paper is that we are able to avoid using the so-called weight function. The weight function in the case of $\mathfrak{g l}_{2}$ can be handled with the help of the functional Bethe ansatz. In the case of $\mathfrak{g l}_{N}, N>2$, the weight function is a much more complicated object and the functional Bethe ansatz is not yet sufficiently developed. We hope that the results of this paper can be used to regularize the weight function.

In the present paper we use the following approach. Let $V=\mathbb{C}^{N}$ be the vector representation of $\mathfrak{g l}_{N}$. We consider the $\mathfrak{g l}_{N}[t]$-module $\mathcal{V}^{S}=\left(V^{\otimes n} \otimes \mathbb{C}\left[z_{1}, \ldots, z_{n}\right]\right)^{S}$ which is a cyclic submodule of the tensor product of evaluation modules with formal evaluation parameters. We show that the Bethe algebra $\mathcal{B}_{\boldsymbol{\lambda}}$ acting on the subspace $\left(\mathcal{V}^{S}\right)_{\boldsymbol{\lambda}}^{\operatorname{sing}}$ of singular vectors of weight $\boldsymbol{\lambda}$ is a free polynomial algebra, see Theorem [5.3, and its representation on $\left(\mathcal{V}^{S}\right)_{\lambda}^{\text {sing }}$ is isomorphic to the regular representation; see Theorem [5.6. To get this result we exploit three observations: a natural identification of the algebra $\mathcal{B}_{\boldsymbol{\lambda}}$ with the algebra of functions on a suitable Schubert cell, the completeness of the Bethe Ansatz for the tensor product of the vector representations at generic evaluation points, and the equality of the graded characters of the Bethe algebra $\mathcal{B}_{\boldsymbol{\lambda}}$ and of the space $\left(\mathcal{V}^{S}\right)_{\boldsymbol{\lambda}}^{\text {sing }}$.

At the second step we specialize the evaluation parameters, and we consider a quotient of the $\mathfrak{g l}_{N}[t]$-module $\mathcal{V}^{S}$ which is isomorphic to the Weyl module. We take the corresponding quotient of the algebra of functions on the Schubert cell and obtain an isomorphism between the scheme-theoretic fiber of the Wronski map and the Bethe algebra $\mathcal{B}_{\boldsymbol{\lambda}, \boldsymbol{a}}$ acting on the subspace of singular vectors of weight $\boldsymbol{\lambda}$ in the Weyl module. We also show that this representation of the algebra $\mathcal{B}_{\boldsymbol{\lambda}, \boldsymbol{a}}$ is isomorphic to the regular representation; see Theorem 5.8. In fact, the algebra $\mathcal{B}_{\boldsymbol{\lambda}, \boldsymbol{a}}$ is Frobenius, so its regular and coregular representations are isomorphic.

Finally, we impose more constraints to descend to the scheme-theoretic intersection of the Schubert varieties on one side and to the Bethe algebra $\mathcal{B}_{\boldsymbol{\Lambda}, \boldsymbol{\lambda}, \boldsymbol{b}}$ acting on a tensor product of evaluation modules with highest weights $\boldsymbol{\lambda}^{(1)}, \ldots, \boldsymbol{\lambda}^{(k)}$ and evaluation parameters $b_{1}, \ldots, b_{k}$ on the other side; see Theorem 5.13 .

The paper is organized as follows. In Section 2, we discuss representations of the current algebra $\mathfrak{g l}_{N}[t]$ and introduce the Bethe algebra $\mathcal{B}$ as a subalgebra of $U\left(\mathfrak{g l}_{N}[t]\right)$. In Section 3, we study the algebra of functions of a Schubert cell and the associated Wronski map. The scheme-theoretic intersection of Schubert cells is considered in Section 4, The central result there is Proposition 4.6, which describes this algebra by generators and relations. We prove the main results of the paper, Theorems 5.3, 5.6, 5.8, and 5.13, in Section 5 Section 6 describes applications.

Other ways to relate the points of intersections of Schubert varieties with invariant vectors in the corresponding tensor products of finite-dimensional irreducible 
$\mathfrak{g l}_{N}$-modules are discussed in [B], Tm. We are not aware of any connection of those works with the method used in the present paper.

\section{Representations of Current algebra $\mathfrak{g l}_{N}[t]$}

2.1. Lie algebra $\mathfrak{g l}_{N}$. Let $e_{i j}, i, j=1, \ldots, N$, be the standard generators of the complex Lie algebra $\mathfrak{g l}_{N}$ satisfying the relations $\left[e_{i j}, e_{s k}\right]=\delta_{j s} e_{i k}-\delta_{i k} e_{s j}$. We identify the Lie algebra $\mathfrak{s l}_{N}$ with the subalgebra in $\mathfrak{g l}_{N}$ generated by the elements $e_{i i}-e_{j j}$ and $e_{i j}$ for $i \neq j, i, j=1, \ldots, N$.

The subalgebra $\mathfrak{z}_{N} \subset \mathfrak{g l}_{N}$ generated by the element $\sum_{i=1}^{N} e_{i i}$ is central. The Lie algebra $\mathfrak{g l}_{N}$ is canonically isomorphic to the direct sum $\mathfrak{s l}_{N} \oplus \mathfrak{z}_{N}$.

Given an $N \times N$ matrix $A$ with possibly noncommuting entries $a_{i j}$, we define its row determinant to be

$$
\operatorname{rdet} A=\sum_{\sigma \in S_{N}}(-1)^{\sigma} a_{1 \sigma(1)} a_{2 \sigma(2)} \ldots a_{N \sigma(N)} .
$$

Let $Z(x)$ be the following polynomial in the variable $x$ with coefficients in $U\left(\mathfrak{g l}_{N}\right)$ :

$$
Z(x)=\operatorname{rdet}\left(\begin{array}{cccc}
x-e_{11} & -<e_{21} & \ldots & -e_{N 1} \\
-e_{12} & x+1-e_{22} & \ldots & -e_{N 2} \\
\cdots & \cdots & \cdots & \cdots \\
-e_{1 N} & -e_{2 N} & \cdots & x+N-1-e_{N N}
\end{array}\right)
$$

The following statement is proved in [HU]; see also Section 2.11 in [MNO].

Theorem 2.1. The coefficients of the polynomial $Z(x)-x^{N}$ are free generators of the center of $U\left(\mathfrak{g l}_{N}\right)$.

Let $M$ be a $\mathfrak{g l}_{N}$-module. A vector $v \in M$ has weight $\boldsymbol{\lambda}=\left(\lambda_{1}, \ldots, \lambda_{N}\right) \in \mathbb{C}^{N}$ if $e_{i i} v=\lambda_{i} v$ for $i=1, \ldots, N$. A vector $v$ is called singular if $e_{i j} v=0$ for $1 \leqslant i<j \leqslant$ $N$. If $v$ is a singular vector of weight $\boldsymbol{\lambda}$, then clearly

$$
Z(x) v=\prod_{i=1}^{N}\left(x-\lambda_{i}+i-1\right) \cdot v .
$$

We denote by $(M)_{\boldsymbol{\lambda}}$ the subspace of $M$ of weight $\boldsymbol{\lambda}$, by $M^{\text {sing }}$ the subspace of $M$ of all singular vectors and by $(M)_{\lambda}^{\text {sing }}$ the subspace of $M$ of all singular vectors of weight $\boldsymbol{\lambda}$.

Denote by $L_{\boldsymbol{\lambda}}$ the irreducible finite-dimensional $\mathfrak{g l}_{N}$-module with highest weight $\boldsymbol{\lambda}$. Any finite-dimensional $\mathfrak{g l}_{N}$-module $M$ is isomorphic to the direct sum $\bigoplus_{\boldsymbol{\lambda}} L_{\boldsymbol{\lambda}} \otimes$ $(M)_{\lambda}^{\text {sing }}$, where the spaces $(M)_{\lambda}^{\text {sing }}$ are considered as trivial $\mathfrak{g l}_{N}$-modules.

The $\mathfrak{g l}_{N}$-module $L_{(1,0, \ldots, 0)}$ is the standard $N$-dimensional vector representation of $\mathfrak{g l}_{N}$. We denote it by $V$. We choose a highest weight vector in $V$ and denote it by $v_{+}$.

$\mathrm{A} \mathfrak{g l}_{N}$-module $M$ is called polynomial if it is isomorphic to a submodule of $V^{\otimes n}$ for some $n$.

A sequence of integers $\boldsymbol{\lambda}=\left(\lambda_{1}, \ldots, \lambda_{N}\right)$ such that $\lambda_{1} \geqslant \lambda_{2} \geqslant \cdots \geqslant \lambda_{N} \geqslant 0$ is called a partition with at most $N$ parts. Set $|\boldsymbol{\lambda}|=\sum_{i=1}^{N} \lambda_{i}$. Then it is said that $\boldsymbol{\lambda}$ is a partition of $|\boldsymbol{\lambda}|$. 
The $\mathfrak{g l}_{N}$-module $V^{\otimes n}$ contains the module $L_{\boldsymbol{\lambda}}$ if and only if $\boldsymbol{\lambda}$ is a partition of $n$ with at most $N$ parts.

For a Lie algebra $\mathfrak{g}$, we denote by $U(\mathfrak{g})$ the universal enveloping algebra of $\mathfrak{g}$.

2.2. Current algebra $\mathfrak{g l}_{N}[t]$. Let $\mathfrak{g l}_{N}[t]=\mathfrak{g l}_{N} \otimes \mathbb{C}[t]$ be the complex Lie algebra of $\mathfrak{g l}_{N}$-valued polynomials with the pointwise commutator. We call it the current algebra. We identify the Lie algebra $\mathfrak{g l}_{N}$ with the subalgebra $\mathfrak{g l}_{N} \otimes 1$ of constant polynomials in $\mathfrak{g l}_{N}[t]$. Hence, any $\mathfrak{g l}_{N}[t]$-module has the canonical structure of a $\mathfrak{g l}_{N}$-module.

The standard generators of $\mathfrak{g l}_{N}[t]$ are $e_{i j} \otimes t^{r}, i, j=1, \ldots, N, r \in \mathbb{Z}_{\geqslant 0}$. They satisfy the relations $\left[e_{i j} \otimes t^{r}, e_{s k} \otimes t^{p}\right]=\delta_{j s} e_{i k} \otimes t^{r+p}-\delta_{i k} e_{s j} \otimes t^{r+p}$.

The subalgebra $\mathfrak{z}_{N}[t] \subset \mathfrak{g l}_{N}[t]$ generated by the elements $\sum_{i=1}^{N} e_{i i} \otimes t^{r}, r \in$ $\mathbb{Z}_{\geqslant 0}$, is central. The Lie algebra $\mathfrak{g l}_{N}[t]$ is canonically isomorphic to the direct sum $\mathfrak{s l}_{N}[t] \oplus \mathfrak{z} N[t]$.

It is convenient to collect elements of $\mathfrak{g l}_{N}[t]$ in generating series of a formal variable $u$. For $g \in \mathfrak{g l}_{N}$, set

$$
g(u)=\sum_{s=0}^{\infty}\left(g \otimes t^{s}\right) u^{-s-1} .
$$

We have $(u-v)\left[e_{i j}(u), e_{s k}(v)\right]=\delta_{j s}\left(e_{i k}(u)-e_{i k}(v)\right)-\delta_{i k}\left(e_{s j}(u)-e_{s j}(v)\right)$.

For each $a \in \mathbb{C}$, there exists an automorphism $\rho_{a}$ of $\mathfrak{g l}_{N}[t], \rho_{a}: g(u) \mapsto g(u-a)$. Given a $\mathfrak{g l}_{N}[t]$-module $M$, we denote by $M(a)$ the pull-back of $M$ through the automorphism $\rho_{a}$. As $\mathfrak{g l}_{N}$-modules, $M$ and $M(a)$ are isomorphic by the identity map.

For any $\mathfrak{g l}_{N}[t]$-modules $L, M$ and any $a \in \mathbb{C}$, the identity map $(L \otimes M)(a) \rightarrow$ $L(a) \otimes M(a)$ is an isomorphism of $\mathfrak{g l}_{N}[t]$-modules.

We have the evaluation homomorphism, ev $: \mathfrak{g l}_{N}[t] \rightarrow \mathfrak{g l}_{N}, \quad$ ev $: g(u) \mapsto g u^{-1}$. Its restriction to the subalgebra $\mathfrak{g l}_{N} \subset \mathfrak{g l}_{N}[t]$ is the identity map. For any $\mathfrak{g l}_{N^{-}}$ module $M$, we denote by the same letter the $\mathfrak{g l}_{N}[t]$-module, obtained by pulling $M$ back through the evaluation homomorphism. For each $a \in \mathbb{C}$, the $\mathfrak{g l}_{N}[t]$-module $M(a)$ is called an evaluation module.

If $b_{1}, \ldots, b_{n}$ are distinct complex numbers and $L_{1}, \ldots, L_{n}$ are irreducible finitedimensional $\mathfrak{g l}_{N}$-modules, then the $\mathfrak{g l}_{N}[t]$-module $\bigotimes_{s=1}^{n} L_{s}\left(b_{s}\right)$ is irreducible.

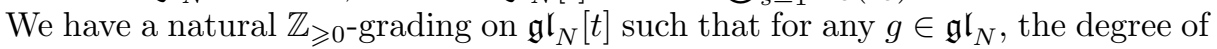
$g \otimes t^{r}$ equals $r$. We set the degree of $u$ to be 1 . Then the series $g(u)$ is homogeneous of degree -1 .

$\mathrm{A} \mathfrak{g l}_{N}[t]$-module is called graded if it has a $\mathbb{Z}_{\geqslant 0}$-grading compatible with the grading of $\mathfrak{g l}_{N}[t]$. Any irreducible graded $\mathfrak{g l}_{N}[t]$-module is isomorphic to an evaluation module $L(0)$ for some irreducible $\mathfrak{g l}_{N}$-module $L$; see $\mathrm{CG}$.

Let $M$ be a $\mathbb{Z}_{\geqslant 0}$-graded space with finite-dimensional homogeneous components. Let $M_{j} \subset M$ be the homogeneous component of degree $j$. We call the formal power series in the variable $q$,

$$
\operatorname{ch}(M)=\sum_{j=0}^{\infty}\left(\operatorname{dim} M_{j}\right) q^{j},
$$

the graded character of $M$. 
2.3. Weyl modules. Let $W_{m}$ be the $\mathfrak{g l}_{N}[t]$-module generated by a vector $v_{m}$ with the defining relations:

$$
\begin{array}{ll}
e_{i i}(u) v_{m}=\delta_{1 i} \frac{m}{u} v_{m}, & i=1, \ldots, N, \\
e_{i j}(u) v_{m}=0, & 1 \leqslant i<j \leqslant N, \\
\left(e_{j i} \otimes 1\right)^{m \delta_{1 i}+1} v_{m}=0, & 1 \leqslant i<j \leqslant N .
\end{array}
$$

As an $\mathfrak{s l}_{N}[t]$-module, the module $W_{m}$ is isomorphic to the Weyl module from [CL], $\mathrm{CP}$, corresponding to the weight $m \omega_{1}$, where $\omega_{1}$ is the first fundamental weight of $\mathfrak{s l}_{N}$. Note that $W_{1}=V(0)$.

Lemma 2.2. The module $W_{m}$ has the following properties.

(i) The module $W_{m}$ has a unique grading such that $W_{m}$ is a graded $\mathfrak{g l}_{N}[t]$ module and such that the degree of $v_{m}$ equals 0 .

(ii) As a $\mathfrak{g l}_{N}$-module, $W_{m}$ is isomorphic to $V^{\otimes m}$.

(iii) $A \mathfrak{g l}_{N}[t]$-module $M$ is an irreducible subquotient of $W_{m}$ if and only if $M$ has the form $L_{\boldsymbol{\lambda}}(0)$, where $\boldsymbol{\lambda}$ is a partition of $m$ with at most $N$ parts.

(iv) For any partition $\boldsymbol{\lambda}$ of $m$ with at most $N$ parts, the graded character of the space $\left(W_{m}\right)_{\lambda}^{\text {sing }}$ is given by

$$
\begin{aligned}
& \operatorname{ch}\left(\left(W_{m}\right)_{\lambda}^{\text {sing }}\right)=\frac{(q)_{m} \prod_{1 \leqslant i<j \leqslant N}\left(1-q^{\lambda_{i}-\lambda_{j}+j-i}\right)}{\prod_{i=1}^{N}(q)_{\lambda_{i}+N-i}} q^{\sum_{i=1}^{N}(i-1) \lambda_{i}}, \\
& \text { where }(q)_{a}=\prod_{j=1}^{a}\left(1-q^{j}\right) .
\end{aligned}
$$

Proof. The first two properties are proved in $\mathrm{CP}$. The third property follows from the first two. The last property is well known; see for example the relation between the character $\operatorname{ch}\left(\left(W_{n}\right)_{\lambda}^{\operatorname{sing}}\right)$ and the Kostka polynomials in [CL, Corollary 1.5.2], and use the formula for the corresponding Kostka polynomial in [M, Example 2, p. 243].

For each $b \in \mathbb{C}$, the $\mathfrak{g l}_{N}[t]$-module $W_{m}(b)$ is cyclic with a cyclic vector $v_{m}$.

Lemma 2.3. The module $W_{m}(b)$ has the following properties.

(i) As a $\mathfrak{g l}_{N}$-module, $W_{m}\left(\right.$ b) is isomorphic to $V^{\otimes m}$.

(ii) $A \mathfrak{g l}_{N}[t]$-module $M$ is an irreducible subquotient of $W_{m}(b)$ if and only if $M$ has the form $L_{\boldsymbol{\lambda}}(b)$, where $\boldsymbol{\lambda}$ is a partition of $m$ with at most $N$ parts.

(iii) For any natural numbers $n_{1}, \ldots, n_{k}$ and distinct complex numbers $b_{1}, \ldots, b_{k}$, the $\mathfrak{g l}_{N}[t]$-module $\bigotimes_{s=1}^{k} W_{n_{s}}\left(b_{s}\right)$ is cyclic with a cyclic vector $\bigotimes_{s=1}^{k} v_{n_{s}}$.

(iv) Let $M$ be a cyclic finite-dimensional $\mathfrak{g l}_{N}[t]$-module with a cyclic vector $v$ satisfying $e_{i j}(u) v=0$ for $1 \leqslant i<j \leqslant N$, and $e_{i i}(u) v=\delta_{1 i}\left(\sum_{s=1}^{k} n_{s}(u-\right.$ $\left.\left.b_{s}\right)^{-1}\right) v$ for $i=1, \ldots, N$. Then there exists a surjective homomorphism of $\mathfrak{g l}_{N}[t]$-modules from $\bigotimes_{s=1}^{k} W_{n_{s}}\left(b_{s}\right)$ to $M$ sending $\bigotimes_{s=1}^{k} v_{n_{s}}$ to $v$.

Proof. The first two properties follow from Lemma 2.2. parts (ii) and (iii). The other two properties are proved in $\mathrm{CP}$.

Given sequences $\boldsymbol{n}=\left(n_{1}, \ldots, n_{k}\right)$ of natural numbers and $\boldsymbol{b}=\left(b_{1}, \ldots, b_{k}\right)$ of distinct complex numbers, we call the $\mathfrak{g l}_{N}[t]$-module $\bigotimes_{s=1}^{k} W_{n_{s}}\left(b_{s}\right)$ the Weyl module associated with $\boldsymbol{n}$ and $\boldsymbol{b}$. 
Corollary 2.4. $A \mathfrak{g l}_{N}[t]$-module $M$ is an irreducible subquotient of $\bigotimes_{s=1}^{k} W_{n_{s}}\left(b_{s}\right)$ if and only if $M$ has the form $\bigotimes_{s=1}^{k} L_{\boldsymbol{\lambda}^{(s)}}\left(b_{s}\right)$, where $\boldsymbol{\lambda}^{(1)}, \ldots, \boldsymbol{\lambda}^{(k)}$ are partitions with at most $N$ parts such that $\left|\boldsymbol{\lambda}^{(s)}\right|=n_{s}, s=1, \ldots, k$.

Proof. The statement follows from part (ii) of Lemma 2.3, irreducibility of $\bigotimes_{s=1}^{k} L_{\boldsymbol{\lambda}^{(s)}}\left(b_{s}\right)$ and the Jordan-Hölder theorem.

Consider the $\mathbb{Z}_{\geqslant 0}$-grading of the vector space $W_{m}$, introduced in Lemma2.2, Let $W_{m}^{j}$ be the homogeneous component of $W_{m}$ of degree $j$ and let $\bar{W}_{m}^{j}=$ $\bigoplus_{r \geqslant j} W_{m}^{r}$. Since the $\mathfrak{g l}_{N}[t]$-module $W_{m}$ is graded and $W_{m}=W_{m}(b)$ as vector spaces, $W_{m}(b)=\bar{W}_{m}^{0} \supset \bar{W}_{m}^{1} \supset \ldots$ is a descending filtration of $\mathfrak{g l}_{N}[t]$-submodules. This filtration induces the structure of the associated graded $\mathfrak{g l}_{N}[t]$-module on the vector space $W_{m}$, which we denote by $\operatorname{gr} W_{m}(b)$.

Lemma 2.5. The $\mathfrak{g l}_{N}[t]$-module gr $W_{m}(b)$ is isomorphic to the evaluation module $\left(V^{\otimes m}\right)(b)$.

The space $\bigotimes_{s=1}^{k} W_{n_{s}}$ has a $\mathbb{Z}_{\geqslant 0}^{k}$-grading, induced by the gradings on the factors and the associated descending $\mathbb{Z}_{\geqslant 0}^{k}$-filtration by the subspaces $\bigotimes_{s=1}^{k} \bar{W}_{n_{s}}^{j_{s}}$. This filtration is compatible with the $\mathfrak{g l}_{N}[t]$-action on the module $\bigotimes_{s=1}^{k} W_{n_{s}}\left(b_{s}\right)$. We denote by $\operatorname{gr}\left(\bigotimes_{s=1}^{k} W_{n_{s}}\left(b_{s}\right)\right)$ the induced structure of the associated graded $\mathfrak{g l}_{N}[t]$ module on the space $\bigotimes_{s=1}^{k} W_{n_{s}}$.

Lemma 2.6. The $\mathfrak{g l}_{N}[t]$-modules $\operatorname{gr}\left(\bigotimes_{s=1}^{k} W_{n_{s}}\left(b_{s}\right)\right)$ and $\bigotimes_{s=1}^{k} \operatorname{gr} W_{n_{s}}\left(b_{s}\right)$ are canonically isomorphic.

2.4. Representations of symmetric group. Let $S_{n}$ be the group of permutations of $n$ elements. We denote by $\mathbb{C}\left[S_{n}\right]$ the regular representation of $S_{n}$. Given an $S_{n}$-module $M$ we denote by $M^{S}$ the subspace of all $S_{n}$-invariant vectors in $M$.

We need the following simple fact.

Lemma 2.7. Let $U$ be a finite-dimensional $S_{n}$-module. Then $\operatorname{dim}\left(U \otimes \mathbb{C}\left[S_{n}\right]\right)^{S}=$ $\operatorname{dim} U$.

The group $S_{n}$ acts on the algebra $\mathbb{C}\left[z_{1}, \ldots, z_{n}\right]$ by permuting the variables. Let $\sigma_{s}(\boldsymbol{z}), s=1, \ldots, n$, be the $s$-th elementary symmetric polynomial in $z_{1}, \ldots, z_{n}$. The algebra of symmetric polynomials $\mathbb{C}\left[z_{1}, \ldots, z_{n}\right]^{S}$ is a free polynomial algebra with generators $\sigma_{1}(\boldsymbol{z}), \ldots, \sigma_{n}(\boldsymbol{z})$. It is well known that the algebra $\mathbb{C}\left[z_{1}, \ldots, z_{n}\right]$ is a free $\mathbb{C}\left[z_{1}, \ldots, z_{n}\right]^{S}$-module of rank $n$ !; see $[\mathrm{M}]$.

Given $\boldsymbol{a}=\left(a_{1}, \ldots, a_{n}\right) \in \mathbb{C}^{n}$, denote by $I_{\boldsymbol{a}} \subset C\left[z_{1}, \ldots, z_{n}\right]$ the ideal generated by the polynomials $\sigma_{s}(\boldsymbol{z})-a_{s}, s=1, \ldots, n$. Clearly, $I_{\boldsymbol{a}}$ is $S_{n}$-invariant.

Lemma 2.8. For any $\boldsymbol{a} \in \mathbb{C}^{n}$, the $S_{n}$-representation $\mathbb{C}\left[z_{1}, \ldots, z_{n}\right] / I_{\boldsymbol{a}}$ is isomorphic to the regular representation $\mathbb{C}\left[S_{n}\right]$.

Proof. Since $\mathbb{C}\left[z_{1}, \ldots, z_{n}\right]$ is a free $\mathbb{C}\left[z_{1}, \ldots, z_{n}\right]^{S}$-module of rank $n$ !, the dimension of the quotient $\mathbb{C}\left[z_{1}, \ldots, z_{n}\right] / I_{\boldsymbol{a}}$ is $n$ ! for all $\boldsymbol{a} \in \mathbb{C}^{n}$. If the polynomial $u^{n}+$ $\sum_{s=1}^{n}(-1)^{s} a_{s} u^{n-s}$ has no multiple roots, the lemma is obvious. For other $\boldsymbol{a}$, the lemma follows by the continuity of the characters.

2.5. $\mathfrak{g l}_{N}[t]$-module $\mathcal{V}^{S}$. Let $\mathcal{V}$ be the space of polynomials in $z_{1}, \ldots, z_{n}$ with coefficients in $V^{\otimes n}$ :

$$
\mathcal{V}=V^{\otimes n} \otimes_{\mathbb{C}} \mathbb{C}\left[z_{1}, \ldots, z_{n}\right] .
$$


The space $V^{\otimes n}$ is embedded in $\mathcal{V}$ as the subspace of constant polynomials.

Abusing notation, for $v \in V^{\otimes n}$ and $p\left(z_{1}, \ldots, z_{n}\right) \in \mathbb{C}\left[z_{1}, \ldots, z_{n}\right]$, we write $p\left(z_{1}, \ldots, z_{n}\right) v$ instead of $v \otimes p\left(z_{1}, \ldots, z_{n}\right)$.

We make the symmetric group $S_{n}$ act on $\mathcal{V}$ by permuting the factors of $V^{\otimes n}$ and the variables $z_{1}, \ldots, z_{n}$ simultaneously,

$$
\begin{aligned}
& \sigma\left(p\left(z_{1}, \ldots, z_{n}\right) v_{1} \otimes \cdots \otimes v_{n}\right) \\
& \quad=p\left(z_{\sigma(1)}, \ldots, z_{\sigma(n)}\right) v_{\sigma^{-1}(1)} \otimes \cdots \otimes v_{\sigma^{-1}(n)}, \quad \sigma \in S_{n} .
\end{aligned}
$$

We denote by $\mathcal{V}^{S}$ the subspace of $S_{n}$-invariants in $\mathcal{V}$.

Lemma 2.9. The space $\mathcal{V}^{S}$ is a free $\mathbb{C}\left[z_{1}, \ldots, z_{n}\right]^{S}$-module of rank $N^{n}$.

Proof. The lemma follows from the fact that $\mathbb{C}\left[z_{1}, \ldots, z_{n}\right]$ is a free $\mathbb{C}\left[z_{1}, \ldots, z_{n}\right]^{S_{-}}$ module. by

We consider the space $\mathcal{V}$ as a $\mathfrak{g l}_{N}[t]$-module with the series $g(u), g \in \mathfrak{g l}_{N}$, acting

$$
g(u)\left(p\left(z_{1}, \ldots, z_{n}\right) v_{1} \otimes \cdots \otimes v_{n}\right)=p\left(z_{1}, \ldots, z_{n}\right) \sum_{s=1}^{n} \frac{v_{1} \otimes \cdots \otimes g v_{s} \otimes \cdots \otimes v_{n}}{u-z_{s}} .
$$

Lemma 2.10. The image of the subalgebra $U\left(\mathfrak{z}_{N}[t]\right) \subset U\left(\mathfrak{g l}_{N}[t]\right)$ in $\operatorname{End}(\mathcal{V})$ coincides with the algebra of operators of multiplication by elements of $\mathbb{C}\left[z_{1}, \ldots, z_{n}\right]^{S}$.

Proof. The element $\sum_{i=1}^{N} e_{i i} \otimes t^{r}$ acts on $\mathcal{V}$ as the operator of multiplication by $\sum_{s=1}^{n} z_{s}^{r}$. The lemma follows.

The $\mathfrak{g l}_{N}[t]$-action on $\mathcal{V}$ commutes with the $S_{n}$-action. Hence, $\mathcal{V}^{S}$ is a $\mathfrak{g l}_{N}[t]$ submodule of $\mathcal{V}$.

The following lemma is contained in $[\underline{K}$. For convenience we supply a proof.

Lemma 2.11. The $\mathfrak{g l}_{N}[t]$-module $\mathcal{V}^{S}$ is cyclic with a cyclic vector $v_{+}^{\otimes n}$.

Proof. Let $v_{1}=v_{+}$and $v_{j}=e_{j 1} v_{+}$for $j=2, \ldots, N$. Then $v_{1}, v_{2}, \ldots, v_{N}$ is a basis of $V$. Given integers $i_{1} \geqslant \cdots \geqslant i_{n} \geqslant 0$ and $j_{1}, \ldots, j_{n} \in\{1, \ldots, N\}$, denote

$$
v(\boldsymbol{i}, \boldsymbol{j})=\sum_{\sigma \in S_{n}} \sigma\left(z_{1}^{i_{1}} \ldots z_{n}^{i_{n}} v_{j_{1}} \otimes \cdots \otimes v_{j_{n}}\right) .
$$

The space $\mathcal{V}^{S}$ is spanned by the elements $v(\boldsymbol{i}, \boldsymbol{j})$ with all possible $\boldsymbol{i}, \boldsymbol{j}$. So to prove the lemma, it is sufficient to show that every element $v(\boldsymbol{i}, \boldsymbol{j})$ belongs to $U\left(\mathfrak{g l}_{N}[t]\right) v_{+}^{\otimes n}$. We prove this by induction on the number $r(\boldsymbol{i}, \boldsymbol{j})$ of indices $s$ such that $i_{s}>0$ and $j_{s}=1$.

If $r(\boldsymbol{i}, \boldsymbol{j})=0$, then $v(\boldsymbol{i}, \boldsymbol{j})=\left(\prod_{s, j_{s} \neq 1} e_{j_{s} 1} \otimes t^{i_{s}}\right) v_{+}^{\otimes n}$.

If $r(\boldsymbol{i}, \boldsymbol{j})>0$, then the difference

$$
v(\boldsymbol{i}, \boldsymbol{j})-\left(\prod_{s, i_{s}>0, j_{s}=1} e_{11} \otimes t^{i_{s}}\right)\left(\prod_{s, j_{s}>1} e_{j_{s} 1} \otimes t^{i_{s}}\right)\left(v_{+}^{\otimes n}\right)
$$

is a linear combination of the elements $v\left(\boldsymbol{i}^{\prime}, \boldsymbol{j}^{\prime}\right)$ with $r\left(\boldsymbol{i}^{\prime}, \boldsymbol{j}^{\prime}\right)<r(\boldsymbol{i}, \boldsymbol{j})$ and belongs to $U\left(\mathfrak{g l}_{N}[t]\right) v_{+}^{\otimes n}$ by the induction hypothesis. Therefore, $v(\boldsymbol{i}, \boldsymbol{j}) \in U\left(\mathfrak{g l}_{N}[t]\right) v_{+}^{\otimes n}$. 
Define the grading on $\mathbb{C}\left[z_{1}, \ldots, z_{n}\right]$ by setting $\operatorname{deg} z_{i}=1$ for all $i=1, \ldots, n$. We define a grading on $\mathcal{V}$ by setting $\operatorname{deg}(v \otimes p)=\operatorname{deg} p$ for any $v \in V^{\otimes n}$ and any $p \in \mathbb{C}\left[z_{1}, \ldots, z_{n}\right]$. The grading on $\mathcal{V}$ induces a natural grading on $\operatorname{End}(\mathcal{V})$.

Lemma 2.12. The $\mathfrak{g l}_{N}[t]$-modules $\mathcal{V}$ and $\mathcal{V}^{S}$ are graded.

Proof. The lemma follows from formula (2.4).

2.6. Weyl modules as quotients of $\mathcal{V}^{S}$. Let $\boldsymbol{a}=\left(a_{1}, \ldots, a_{n}\right) \in \mathbb{C}^{n}$ be a sequence of complex numbers and let $I_{\boldsymbol{a}} \subset \mathbb{C}\left[z_{1}, \ldots, z_{n}\right]$ be the ideal defined in Section 2.4 Define

$$
I_{\boldsymbol{a}}^{\mathcal{V}}=\left(V^{\otimes n} \otimes I_{\boldsymbol{a}}\right) \cap \mathcal{V}^{S}
$$

Clearly, $I_{\boldsymbol{a}}^{\mathcal{V}}$ is a $\mathfrak{g l}_{N}[t]$-submodule of $\mathcal{V}^{S}$ and a free $\mathbb{C}\left[z_{1}, \ldots, z_{n}\right]^{S}$-module.

Define distinct complex numbers $b_{1}, \ldots, b_{k}$ and natural numbers $n_{1}, \ldots, n_{k}$ by the relation

$$
\prod_{s=1}^{k}\left(u-b_{s}\right)^{n_{s}}=u^{n}+\sum_{j=1}^{n}(-1)^{j} a_{j} u^{n-j} .
$$

Clearly, $\sum_{s=1}^{k} n_{s}=n$.

Lemma 2.13. The $\mathfrak{g l}_{N}[t]$-module $\mathcal{V}^{S} / I_{\boldsymbol{a}}^{\mathcal{V}}$ is isomorphic to the Weyl module $\bigotimes_{s=1}^{k} W_{n_{s}}\left(b_{s}\right)$.

Proof. Let $\overline{v_{+}^{\otimes n}}$ be the image of the vector $v_{+}^{\otimes n}$ in the quotient space $\mathcal{V}^{S} / I_{\boldsymbol{a}}^{\mathcal{V}}$. Then by Lemma 2.11, the quotient space $\mathcal{V}^{S} / I_{\boldsymbol{a}}^{\mathcal{V}}$ is a cyclic $\mathfrak{g l}_{N}[t]$-module with a cyclic vector $\overline{v_{+}^{\otimes n}}$, and

$$
e_{i i}(u) \overline{v_{+}^{\otimes n}}=\sum_{s=1}^{k} \delta_{1 i} \frac{n_{s}}{u-b_{s}} \overline{v_{+}^{\otimes n}} .
$$

By part (iv) of Lemma 2.3, there exists a surjective homomorphism

$$
\bigotimes_{s=1}^{k} W_{n_{s}}\left(b_{s}\right) \rightarrow \mathcal{V}^{S} / I_{\boldsymbol{a}}^{\mathcal{V}}
$$

In addition,

$$
\begin{aligned}
\operatorname{dim}\left(\mathcal{V}^{S} / I_{\boldsymbol{a}}^{\mathcal{V}}\right)=\operatorname{dim}\left(\mathcal{V} / I_{\boldsymbol{a}}^{\mathcal{V}}\right)^{S} & =\operatorname{dim}\left(V^{\otimes n} \otimes \mathbb{C}\left[z_{1}, \ldots, z_{n}\right] / I_{\boldsymbol{a}}\right)^{S} \\
& =\operatorname{dim} V^{\otimes n}=\operatorname{dim}\left(\bigotimes_{s=1}^{k} W_{n_{s}}\left(b_{s}\right)\right),
\end{aligned}
$$

where we used Lemmas 2.8 and 2.7 for the next to the last equality. Therefore, the surjective homomorphism (2.7) is an isomorphism.

Lemma 2.14. We have

$$
I_{\boldsymbol{a}}^{\mathcal{V}}=\left(I_{\boldsymbol{a}} \mathcal{V}^{S}\right) \cap \mathcal{V}^{S}
$$

Proof. We have $\left(\left(I_{\boldsymbol{a}} \mathcal{V}^{S}\right) \cap \mathcal{V}^{S}\right) \subset I_{\boldsymbol{a}}^{\mathcal{V}} \subset \mathcal{V}^{S}$. By Lemmas 2.9 and 2.13 we have

$$
\operatorname{dim} \mathcal{V}^{S} /\left(\left(I_{\boldsymbol{a}} \mathcal{V}^{S}\right) \cap \mathcal{V}^{S}\right)=N^{n}=\operatorname{dim} \mathcal{V}^{S} / I_{\boldsymbol{a}}^{\mathcal{V}}
$$

The lemma follows. 
2.7. Bethe algebra. Let $\partial$ be the operator of formal differentiation in the variable $u$. Define the universal differential operator $\mathcal{D}^{\mathcal{B}}$ by

$$
\mathcal{D}^{\mathcal{B}}=\operatorname{rdet}\left(\begin{array}{cccc}
\partial-e_{11}(u) & -e_{21}(u) & \ldots & -e_{N 1}(u) \\
-e_{12}(u) & \partial-e_{22}(u) & \ldots & -e_{N 2}(u) \\
\ldots & \ldots & \ldots & \ldots \\
-e_{1 N}(u) & -e_{2 N}(u) & \ldots & \partial-e_{N N}(u)
\end{array}\right) .
$$

It is a differential operator in the variable $u$, whose coefficients are formal power series in $u^{-1}$ with coefficients in $U\left(\mathfrak{g l}_{N}[t]\right)$,

where

$$
\mathcal{D}^{\mathcal{B}}=\partial^{N}+\sum_{i=1}^{N} B_{i}(u) \partial^{N-i}
$$

$$
B_{i}(u)=\sum_{j=1}^{\infty} B_{i j} u^{-j}
$$

and $B_{i j} \in U\left(\mathfrak{g l}_{N}[t]\right), i=1, \ldots, N, j \in \mathbb{Z}_{>0}$. Clearly, $B_{i j}=0$ for $j<i$.

Lemma 2.15. We have

$$
B_{1}(u)=-\sum_{i=1}^{N} e_{i i}(u)
$$

and

$$
\sum_{i=0}^{N} B_{i i} \prod_{j=0}^{N-i-1}(\alpha-j)=Z(\alpha-N+1),
$$

where $\alpha$ is a formal variable, $B_{00}=1$, and $Z(x)$ is given by formula (2.2).

Proof. The lemma is proved by a straightforward calculation.

Lemma 2.16. For any $i, j$, the element $B_{i j} \in U\left(\mathfrak{g l}_{N}[t]\right)$ is a homogeneous element of degree $j-i$. The series $B_{i}(u)$ is a homogeneous series of degree $-i$.

Proof. We declare the degree of $\partial$ to be -1 . Then $\mathcal{D}^{\mathcal{B}}$ is homogeneous of degree $-N$. The lemma follows.

We call the unital subalgebra of $U\left(\mathfrak{g l}_{N}[t]\right)$ generated by $B_{i j}, i=1, \ldots, N, j \in$ $\mathbb{Z}_{\geqslant i}$, the Bethe algebra and denote it by $\mathcal{B}$.

The next statement is established in [T. A polished proof can be found in MTV1.

Theorem 2.17. The algebra $\mathcal{B}$ is commutative. The algebra $\mathcal{B}$ commutes with the subalgebra $U\left(\mathfrak{g l}_{N}\right) \subset U\left(\mathfrak{g l}_{N}[t]\right)$.

As a subalgebra of $U\left(\mathfrak{g l}_{N}[t]\right)$, the algebra $\mathcal{B}$ acts on any $\mathfrak{g l}_{N}[t]$-module $M$. If $K \subset M$ is a $\mathcal{B}$-invariant subspace, then we call the image of $\mathcal{B}$ in $\operatorname{End}(K)$ the Bethe algebra associated with $K$. Since $\mathcal{B}$ commutes with $U\left(\mathfrak{g l}_{N}\right)$, it preserves the subspace of singular vectors $M^{\text {sing }}$ as well as weight subspaces of $M$. Therefore, the subspace $(M)_{\lambda}^{\text {sing }}$ is $\mathcal{B}$-invariant for any weight $\boldsymbol{\lambda}$.

Let $\boldsymbol{\lambda}$ be a partition with at most $N$ parts. Let $n=|\boldsymbol{\lambda}|$ and $\left(a_{1}, \ldots, a_{n}\right) \in \mathbb{C}^{n}$. Define integers $k, n_{1}, \ldots, n_{k}$ and distinct complex numbers $b_{1}, \ldots, b_{k}$ by (2.6). Let $\boldsymbol{\Lambda}=\left(\boldsymbol{\lambda}^{(1)}, \ldots, \boldsymbol{\lambda}^{(k)}\right)$ be a sequence of partitions with at most $N$ parts such that $\left|\boldsymbol{\lambda}_{s}\right|=n_{s}$. 
In what follows we study the action of the Bethe algebra $\mathcal{B}$ on the following $\mathcal{B}$-modules:

$$
\begin{aligned}
& \mathcal{M}_{\boldsymbol{\lambda}}=\left(\mathcal{V}^{S}\right)_{\boldsymbol{\lambda}}^{\text {sing }}, \\
& \mathcal{M}_{\boldsymbol{\lambda}, \boldsymbol{a}}=\left(\bigotimes_{s=1}^{k} W_{n_{s}}\left(b_{s}\right)\right)_{\boldsymbol{\lambda}}^{\text {sing }}, \\
& \mathcal{M}_{\boldsymbol{\Lambda}, \boldsymbol{\lambda}, \boldsymbol{b}}=\left(\bigotimes_{s=1}^{k} L_{\boldsymbol{\lambda}^{(s)}}\left(b_{s}\right)\right)_{\boldsymbol{\lambda}}^{\text {sing }} .
\end{aligned}
$$

The $\mathcal{B}$-modules $\mathcal{M}_{\boldsymbol{\lambda}, \boldsymbol{a}}$ and $\mathcal{M}_{\boldsymbol{\Lambda}, \boldsymbol{\lambda}, \boldsymbol{b}}$ are defined by formula (2.12) up to isomorphism.

We denote the Bethe algebras associated with $\mathcal{M}_{\boldsymbol{\lambda}}, \mathcal{M}_{\boldsymbol{\lambda}, \boldsymbol{a}}, \mathcal{M}_{\boldsymbol{\Lambda}, \boldsymbol{\lambda}, \boldsymbol{b}}$ by $\mathcal{B}_{\boldsymbol{\lambda}}$, $\mathcal{B}_{\boldsymbol{\lambda}, \boldsymbol{a}}, \mathcal{B}_{\boldsymbol{\Lambda}, \boldsymbol{\lambda}, \boldsymbol{b}}$, respectively.

\section{Functions on Schubert CELl And Wronski map}

3.1. Functions on Schubert cell $\Omega_{\bar{\lambda}}(\infty)$. Let $N, d \in \mathbb{Z}_{>0}, N \leqslant d$. Let $\mathbb{C}_{d}[u]$ be the space of polynomials in $u$ of degree less than $d$. We have $\operatorname{dim} \mathbb{C}_{d}[u]=d$. Let $\operatorname{Gr}(N, d)$ be the Grassmannian of all $N$-dimensional subspaces in $\mathbb{C}[d]$. The Grassmannian $\operatorname{Gr}(N, d)$ is a smooth projective variety of dimension $N(d-N)$.

For a complete flag $\mathcal{F}=\left\{0 \subset F_{1} \subset F_{2} \subset \cdots \subset F_{d}=\mathbb{C}_{d}[u]\right\}$ and a partition $\boldsymbol{\lambda}=\left(\lambda_{1}, \ldots, \lambda_{N}\right)$ such that $\lambda_{1} \leqslant d-N$, the $\operatorname{Schubert}$ cell $\Omega_{\boldsymbol{\lambda}}(\mathcal{F}) \subset \operatorname{Gr}(N, d)$ is given by

$$
\begin{aligned}
\Omega_{\boldsymbol{\lambda}}(\mathcal{F})=\{X \in \operatorname{Gr}(N, d) \mid & \operatorname{dim}\left(X \cap F_{d-j-\lambda_{j}}\right)=N-j, \\
& \left.\operatorname{dim}\left(X \cap F_{d-j-\lambda_{j}-1}\right)=N-j-1\right\} .
\end{aligned}
$$

We have codim $\Omega_{\boldsymbol{\lambda}}(\mathcal{F})=|\boldsymbol{\lambda}|$.

The Schubert cell decomposition associated to a complete flag $\mathcal{F}$, see for example $\mathrm{GH}$, is given by

$$
\operatorname{Gr}(N, d)=\bigsqcup_{\boldsymbol{\lambda}, \lambda_{1} \leqslant d-N} \Omega_{\boldsymbol{\lambda}}(\mathcal{F})
$$

Given a partition $\boldsymbol{\lambda}=\left(\lambda_{1}, \ldots, \lambda_{N}\right)$ such that $\lambda_{1} \leqslant d-N$, introduce a set

$$
P=\left\{d_{1}, d_{2}, \ldots, d_{N}\right\}, \quad d_{i}=\lambda_{i}+N-i
$$

and a new partition

$$
\bar{\lambda}=\left(d-N-\lambda_{N}, d-N-\lambda_{N-1}, \ldots, d-N-\lambda_{1}\right) .
$$

Then $d_{1}>d_{2}>\cdots>d_{N}$ and

$$
|\boldsymbol{\lambda}|=N(d-N)-|\overline{\boldsymbol{\lambda}}|=\sum_{i=1}^{N} d_{i}-N(N-1) / 2 .
$$

Let $\mathcal{F}(\infty)$ be the complete flag given by

$$
\mathcal{F}(\infty)=\left\{0 \subset \mathbb{C}_{1}[u] \subset \mathbb{C}_{2}[u] \subset \cdots \subset \mathbb{C}_{d}[u]\right\}
$$

We denote the Schubert cell $\Omega_{\bar{\lambda}}(\mathcal{F}(\infty))$ by $\Omega_{\bar{\lambda}}(\infty)$. We have $\operatorname{dim} \Omega_{\bar{\lambda}}(\infty)=|\boldsymbol{\lambda}|$.

The Schubert cell $\Omega_{\bar{\lambda}}(\infty) \subset \operatorname{Gr}(N, d)$ consists of $N$-dimensional subspaces $X \subset$ $\mathbb{C}_{d}[u]$ which have a basis $\left\{f_{1}(u), \ldots, f_{N}(u)\right\}$ of the form

$$
f_{i}(u)=u^{d_{i}}+\sum_{j=1, d_{i}-j \notin P}^{d_{i}} f_{i j} u^{d_{i}-j} .
$$


For a given subspace $X \in \Omega_{\bar{\lambda}}(\infty)$, such a basis is unique.

Let $\mathcal{O}_{\boldsymbol{\lambda}}$ be the algebra of regular functions on $\Omega_{\bar{\lambda}}(\infty)$. The cell $\Omega_{\bar{\lambda}}(\infty)$ is an affine space of dimension $|\boldsymbol{\lambda}|$ with coordinate functions $f_{i j}$. Therefore, the algebra $\mathcal{O}_{\lambda}$ is a free polynomial algebra in the variables $f_{i j}$,

$$
\mathcal{O}_{\boldsymbol{\lambda}}=\mathbb{C}\left[f_{i j}, i=1, \ldots, N, j=1, \ldots, d_{i}, d_{i}-j \notin P\right] .
$$

We often regard the polynomials $f_{i}(u), i=1, \ldots, N$, as generating functions for the generators $f_{i j}$ of $\mathcal{O}_{\lambda}$.

Recall that the degree of $u$ is one. Define a grading on the algebra $\mathcal{O}_{\boldsymbol{\lambda}}$ by setting the degree of the generator $f_{i j}$ to be $j$. Then the generating function $f_{i}(u)$ is homogeneous of degree $d_{i}$.

Lemma 3.1. The graded character of $\mathcal{O}_{\boldsymbol{\lambda}}$ is given by the formula

$$
\operatorname{ch}\left(\mathcal{O}_{\boldsymbol{\lambda}}\right)=\frac{\prod_{1 \leqslant i<j \leqslant N}\left(1-q^{d_{i}-d_{j}}\right)}{\prod_{i=1}^{N}(q)_{d_{i}}} .
$$

3.2. Another realization of $\mathcal{O}_{\boldsymbol{\lambda}}$. For $g_{1}, \ldots, g_{N} \in \mathbb{C}[u]$, denote by $\operatorname{Wr}\left(g_{1}(u), \ldots\right.$, $\left.g_{N}(u)\right)$ the Wronskian,

$$
\mathrm{Wr}\left(g_{1}(u), \ldots, g_{N}(u)\right)=\operatorname{det}\left(\begin{array}{cccc}
g_{1}(u) & g_{1}^{\prime}(u) & \ldots & g_{1}^{(N-1)}(u) \\
g_{2}(u) & g_{2}^{\prime}(u) & \ldots & g_{2}^{(N-1)}(u) \\
\ldots & \ldots & \ldots & \ldots \\
g_{N}(u) & g_{N}^{\prime}(u) & \ldots & g_{N}^{(N-1)}(u)
\end{array}\right) .
$$

Let $f_{i}(u), i=1, \ldots, N$, be the generating functions given by (3.2). We have

$$
\operatorname{Wr}\left(f_{1}(u), \ldots, f_{N}(u)\right)=\prod_{1 \leqslant i<j \leqslant N}\left(d_{j}-d_{i}\right)\left(u^{n}+\sum_{s=1}^{n}(-1)^{s} \Sigma_{s} u^{n-s}\right),
$$

where $\Sigma_{1}, \ldots, \Sigma_{n}$ are elements of $\mathcal{O}_{\boldsymbol{\lambda}}$. Define the differential operator $\mathcal{D}_{\boldsymbol{\lambda}}^{\mathcal{O}}$ by

$$
\mathcal{D}_{\lambda}^{\mathcal{O}}=\frac{1}{\operatorname{Wr}\left(f_{1}(u), \ldots, f_{N}(u)\right)} \operatorname{rdet}\left(\begin{array}{cccc}
f_{1}(u) & f_{1}^{\prime}(u) & \ldots & f_{1}^{(N)}(u) \\
f_{2}(u) & f_{2}^{\prime}(u) & \ldots & f_{2}^{(N)}(u) \\
\ldots & \ldots & \ldots & \ldots \\
1 & \partial & \ldots & \partial^{N}
\end{array}\right) .
$$

It is a differential operator in the variable $u$, whose coefficients are rational functions with coefficients in $\mathcal{O}_{\boldsymbol{\lambda}}$,

$$
\mathcal{D}_{\boldsymbol{\lambda}}^{\mathcal{O}}=\partial^{N}+\sum_{i=1}^{N} F_{i}(u) \partial^{N-i} .
$$

The top coefficient of the Wronskian $\operatorname{Wr}\left(f_{1}(u), \ldots, f_{N}(u)\right)$ is a constant. Therefore, we can write

$$
F_{i}(u)=\sum_{j=1}^{\infty} F_{i j} u^{-j}
$$

where $F_{i j} \in \mathcal{O}_{\boldsymbol{\lambda}}, i=1, \ldots, N, j \in \mathbb{Z}_{>0}$. Clearly, $F_{i j}=0$ for $j<i$.

Lemma 3.2. For any $i, j$, the element $F_{i j} \in \mathcal{O}_{\boldsymbol{\lambda}}$ is a homogeneous element of degree $j-i$. The series $F_{i}(u)$ is a homogeneous series of degree $-i$.

Proof. Recall that $\partial$ has degree -1 . So, the operator $\mathcal{D}_{\lambda}^{\mathcal{O}}$ is homogeneous of degree $-N$. The lemma follows. 
Define the indicial polynomial of the operator $\mathcal{D}_{\boldsymbol{\lambda}}^{\mathcal{O}}$ at infinity by

$$
\chi(\alpha)=\sum_{i=0}^{N} F_{i i} \prod_{j=0}^{N-i-1}(\alpha-j),
$$

where $F_{00}=1$.

Lemma 3.3. We have

$$
\chi(\alpha)=\prod_{i=1}^{N}\left(\alpha-d_{i}\right) .
$$

Proof. The coefficient of $u^{d_{i}-N}$ of the series $\mathcal{D}_{\lambda}^{\mathcal{O}} f_{i}(u)$ equals $\chi\left(d_{i}\right)$. On the other hand, we have that $\mathcal{D}_{\lambda}^{\mathcal{O}} f_{i}(u)=0$, and therefore, $\chi\left(d_{i}\right)=0$ for all $i=1, \ldots, N$. Since $\operatorname{deg} \chi=N$, the lemma follows.

Lemma 3.4. The functions $F_{i j} \in \mathcal{O}_{\boldsymbol{\lambda}}, i=1, \ldots, N, j=i+1, i+2, \ldots$, generate the algebra $\mathcal{O}_{\lambda}$.

Proof. The coefficient of $u^{d_{i}-N-j}$ of the series $\mathcal{D}_{\lambda}^{\mathcal{O}} f_{i}(u)$ has the form

$$
\chi\left(d_{i}-j\right) f_{i j}+\ldots,
$$

where the dots denote the terms which contain the elements $F_{k l}$ and $f_{i s}$ with $s<j$ only. Since $\mathcal{D}_{\boldsymbol{\lambda}}^{\mathcal{O}} f_{i}(u)=0$ and $\chi\left(d_{i}-j\right) \neq 0$, see (3.3), we can express recursively the elements $f_{i j}$ via the elements $F_{k l}$ starting with $j=1$ and then increasing the second index $j$.

3.3. Frobenius algebras. In this section, we recall some simple facts from commutative algebra. We use the word algebra for an associative unital algebra over $\mathbb{C}$.

Let $A$ be a commutative algebra. The algebra $A$ considered as an $A$-module is called the regular representation of $A$. The dual space $A^{*}$ is naturally an $A$-module, which is called the coregular representation.

Clearly, the image of $A$ in $\operatorname{End}(A)$ for the regular representation is a maximal commutative subalgebra. If $A$ is finite-dimensional, then the image of $A$ in $\operatorname{End}\left(A^{*}\right)$ for the coregular representation is a maximal commutative subalgebra as well.

If $M$ is an $A$-module and $v \in M$ is an eigenvector of the $A$-action on $M$ with eigenvalue $\xi_{v} \in A^{*}$, that is, $a v=\xi_{v}(a) v$ for any $a \in A$, then $\xi_{v}$ is a character of $A$, that is, $\xi_{v}(a b)=\xi_{v}(a) \xi_{v}(b)$.

If an element $v \in A^{*}$ is an eigenvector of the coregular action of $A$, then $v$ is proportional to the character $\xi_{v}$. Moreover, each character $\xi \in A^{*}$ is an eigenvector of the coregular action of $A$, and the corresponding eigenvalue equals $\xi$.

A nonzero element $\xi \in A^{*}$ is proportional to a character if and only if $\operatorname{ker} \xi \subset A$ is an ideal. Clearly, $A / \operatorname{ker} \xi \simeq \mathbb{C}$. On the other hand, if $\mathfrak{m} \subset A$ is an ideal such that $A / \mathfrak{m} \simeq \mathbb{C}$, then $\mathfrak{m}$ is a maximal proper ideal and $\mathfrak{m}=\operatorname{ker} \zeta$ for some character $\zeta$.

A commutative algebra $A$ is called local if it has a unique ideal $\mathfrak{m}$ such that $A / \mathfrak{m} \simeq \mathbb{C}$. In other words, a commutative algebra $A$ is local if it has a unique character. Any proper ideal of the local finite-dimensional algebra $A$ is contained in the ideal $\mathfrak{m}$.

It is known that any finite-dimensional commutative algebra $A$ is isomorphic to a direct sum of local algebras, and the local summands are in bijection with characters of $A$. 
Let $A$ be a commutative algebra. A bilinear form $():, A \otimes A \rightarrow \mathbb{C}$ is called invariant if $(a b, c)=(a, b c)$ for all $a, b, c \in A$.

A finite-dimensional commutative algebra $A$ which admits an invariant nondegenerate symmetric bilinear form $():, A \otimes A \rightarrow \mathbb{C}$ is called a Frobenius algebra. It is easy to see that distinct local summands of a Frobenius algebra are orthogonal.

The following properties of Frobenius algebras will be useful.

Lemma 3.5. A finite direct sum of Frobenius algebras is a Frobenius algebra.

Proof. Fix invariant nondegenerate symmetric bilinear forms on the summands and define a bilinear form on the direct sum to be the direct sum of the forms of the summands. The obtained form is clearly nondegenerate, symmetric and invariant.

Let $A$ be a Frobenius algebra. Let $I \subset A$ be a subspace. Denote by $I^{\perp} \subset A$ the orthogonal complement to $I$. Then $\operatorname{dim} I+\operatorname{dim} I^{\perp}=\operatorname{dim} A$, and the subspace $I$ is an ideal if and only if $I^{\perp}$ is an ideal.

Let $A_{0}$ be a local Frobenius algebra with maximal ideal $\mathfrak{m} \subset A_{0}$. Then $\mathfrak{m}^{\perp}$ is a one-dimensional ideal. Let $m^{\perp} \in \mathfrak{m}^{\perp}$ be an element such that $\left(1, m^{\perp}\right)=1$.

Lemma 3.6. Any nonzero ideal $I \subset A_{0}$ contains $\mathfrak{m}^{\perp}$.

Proof. Let $I \subset A_{0}$ be a nonzero ideal. Then $I^{\perp}$ is also an ideal and $I^{\perp} \neq A_{0}$. Therefore, $I^{\perp} \subset \mathfrak{m}$ and $\mathfrak{m}^{\perp} \subset I$.

For a subset $I \subset A$ define its annihilator as Ann $I=\{a \in A \mid a I=0\}$. The annihilator Ann $I$ is an ideal.

Lemma 3.7. Let $A$ be a Frobenius algebra and $I \subset A$ an ideal. Then $A n n I=I^{\perp}$. In particular, $\operatorname{dim} I+\operatorname{dim} A \operatorname{Ann} I=\operatorname{dim} A$.

Proof. Since every ideal in a finite-dimensional commutative algebra is a direct sum of ideals in its local summands, it is sufficient to prove the lemma for the case of a local Frobenius algebra.

Let $A$ be local. If $a \in I$ and $b \in \operatorname{Ann} I$, then $(a, b)=(a b, 1)=0$. Therefore, Ann $I \subset I^{\perp}$.

If $b \in I^{\perp}$, then $b I \subset I^{\perp}$ is an ideal. If $b I \neq 0$, then it contains $\mathfrak{m}^{\perp}$, see Lemma 3.6, and there exists $a \in I$ such that $a b=m^{\perp}$. Hence, $0=(a, b)=(1, a b)=\left(1, m^{\perp}\right)=$ 1 , which is a contradiction. Therefore, $b I=0$ and $I^{\perp} \subset$ Ann $I$.

For any ideal $I \subset A$, the regular action of $A$ on itself induces an action of $A / I$ on Ann $I$.

Lemma 3.8. The $A / I$-module Ann $I$ is isomorphic to the coregular representation of $A / I$. In particular, the image of $A / I$ in $\operatorname{End}(\operatorname{Ann} I)$ is a maximal commutative subalgebra.

Proof. The form (,) gives a natural isomorphism of the ideal Ann $I$ and the dual space $(A / I)^{*}$, which identifies the action of $A / I$ on Ann $I$ with the coregular action of $A / I$ on $(A / I)^{*}$.

Let $P_{1}, \ldots, P_{m}$ be polynomials in the variables $x_{1}, \ldots, x_{m}$. Denote by $I$ the ideal in $\mathbb{C}\left[x_{1}, \ldots, x_{m}\right]$ generated by $P_{1}, \ldots, P_{m}$.

Lemma 3.9. If the algebra $\mathbb{C}\left[x_{1}, \ldots, x_{m}\right] / I$ is nonzero and finite-dimensional, then it is a Frobenius algebra. 
Proof. For $F, G \in \mathbb{C}\left[x_{1}, \ldots, x_{m}\right]$, define the residue form

$$
\operatorname{Res}(F, G)=\frac{1}{(2 \pi i)^{m}} \int_{\Gamma} \frac{F G d x_{1} \wedge \cdots \wedge d x_{m}}{\prod_{s=1}^{m} P_{s}\left(x_{1}, \ldots, x_{m}\right)},
$$

where $\Gamma=\left\{\left(x_{1}, \ldots, x_{m}\right)|| P_{s}\left(x_{1}, \ldots, x_{m}\right) \mid=\varepsilon_{s}\right\}$ is the real $m$-cycle oriented by the condition

$$
d \arg P_{1}\left(x_{1}, \ldots, x_{m}\right) \wedge \cdots \wedge d \arg P_{m}\left(x_{1}, \ldots, x_{m}\right) \geqslant 0
$$

and $\varepsilon_{s}$ are sufficiently small positive real numbers. The residue form Res descends to a nondegenerate bilinear form on $\mathbb{C}\left[x_{1}, \ldots, x_{m}\right] / I$; see $[\mathrm{GH}]$.

The last lemma has the following generalization. Let $\mathbb{C}_{T}\left(x_{1}, \ldots, x_{m}\right)$ be the algebra of rational functions in $x_{1}, \ldots, x_{m}$, regular at points of a nonempty set $T \subset \mathbb{C}^{m}$. Denote by $I_{T}$ the ideal in $\mathbb{C}_{T}\left(x_{1}, \ldots, x_{m}\right)$ generated by $P_{1}, \ldots, P_{m}$.

Lemma 3.10. Assume that the solution set to the system of equations $P_{i}\left(x_{1}, \ldots\right.$, $\left.x_{m}\right)=0, i=1, \ldots, m$, is finite and lies in $T$. Then the algebra $\mathbb{C}_{T}\left(x_{1}, \ldots, x_{m}\right) / I_{T}$ is a Frobenius algebra.

3.4. Wronski map. Let $X$ be a point of $\operatorname{Gr}(N, d)$. The Wronskian of a basis of the subspace $X$ does not depend on the choice of the basis up to multiplication by a nonzero number. We call the monic polynomial representing the Wronskian the Wronskian of $X$ and denote it by $\mathrm{Wr}_{X}(u)$.

Fix a partition $\boldsymbol{\lambda}$ and denote $n=|\boldsymbol{\lambda}|$. The partition $\overline{\boldsymbol{\lambda}}$ is given by (3.1). If $X \in \Omega_{\bar{\lambda}}(\infty)$, then $\operatorname{deg} \operatorname{Wr}_{X}(u)=n$.

Define the Wronski map

$$
\mathrm{Wr}_{\boldsymbol{\lambda}}: \Omega_{\bar{\lambda}}(\infty) \rightarrow \mathbb{C}^{n}
$$

by sending $X \in \Omega_{\overline{\boldsymbol{\lambda}}}(\infty)$ to $\boldsymbol{a}=\left(a_{1}, \ldots, a_{n}\right)$ if $\mathrm{Wr}_{X}(u)=u^{n}+\sum_{s=1}^{n}(-1)^{s} a_{s} u^{n-s}$.

For $\boldsymbol{a} \in \mathbb{C}^{n}$, let $I_{\boldsymbol{\lambda}, \boldsymbol{a}}^{\mathcal{O}}$ be the ideal in $\mathcal{O}_{\boldsymbol{\lambda}}$ generated by the elements $\Sigma_{s}-a_{s}$, $s=1, \ldots, n$, where $\Sigma_{1}, \ldots, \Sigma_{n}$ are defined by (3.4). Let

$$
\mathcal{O}_{\lambda, a}=\mathcal{O}_{\lambda} / I_{\lambda, a}^{\mathcal{O}}
$$

be the quotient algebra. The algebra $\mathcal{O}_{\boldsymbol{\lambda}, \boldsymbol{a}}$ is the scheme-theoretic fiber of the Wronski map. We call it the algebra of functions on the preimage $\operatorname{Wr}_{\boldsymbol{\lambda}}^{-1}(\boldsymbol{a})$.

Lemma 3.11. The algebra $\mathcal{O}_{\boldsymbol{\lambda}, \boldsymbol{a}}$ is a finite-dimensional Frobenius algebra and $\operatorname{dim}_{\mathbb{C}} \mathcal{O}_{\boldsymbol{\lambda}, \boldsymbol{a}}$ does not depend on $\boldsymbol{a}$.

Proof. It is easy to show that the set $\mathrm{Wr}_{\boldsymbol{\lambda}}^{-1}(\boldsymbol{a})$ is finite; see for example Propositions 3.1 and 4.2 in MTV5. This implies that $\mathcal{O}_{\boldsymbol{\lambda}, \boldsymbol{a}}$ is finite-dimensional and the fact that $\mathcal{O}_{\boldsymbol{\lambda}, \boldsymbol{a}}$ is a direct sum of local algebras; see for example $\mathrm{HP}$. The dimension of $\mathcal{O}_{\boldsymbol{\lambda}, \boldsymbol{a}}$ is the degree of the Wronski map and the local summands correspond to the points of the set $\mathrm{Wr}_{\boldsymbol{\lambda}}^{-1}(\boldsymbol{a})$.

The algebra $\mathcal{O}_{\boldsymbol{\lambda}, \boldsymbol{a}}$ is Frobenius by Lemma 3.9.

Remark. Let $\mathcal{O}_{\lambda}^{S} \subset \mathcal{O}_{\boldsymbol{\lambda}}$ be the subalgebra generated by $\Sigma_{1}, \ldots, \Sigma_{n}$. Since these elements are homogeneous, the subalgebra $\mathcal{O}_{\lambda}^{S}$ is graded. Using the grading and Lemmas 3.11, 2.2, it is easy to see that $\mathcal{O}_{\boldsymbol{\lambda}}$ is a free $\mathcal{O}_{\lambda}^{S}$-module of $\operatorname{rank} \operatorname{dim}\left(V^{\otimes n}\right)_{\lambda}^{\operatorname{sing}}$. 


\section{Functions on intersection of Schubert Cells}

4.1. Functions on $\Omega_{\Lambda, \bar{\lambda}, b}$. For $b \in \mathbb{C}$, consider the complete flag

$$
\mathcal{F}(b)=\left\{0 \subset(u-b)^{d-1} \mathbb{C}_{1}[u] \subset(u-b)^{d-2} \mathbb{C}_{2}[u] \subset \cdots \subset \mathbb{C}_{d}[u]\right\}
$$

We denote the Schubert cell $\Omega_{\boldsymbol{\mu}}(\mathcal{F}(b))$ corresponding to the flag $\mathcal{F}(b)$ and a partition $\boldsymbol{\mu}$ with at most $N$ parts by $\Omega_{\boldsymbol{\mu}}(b)$.

Let $\boldsymbol{\Lambda}=\left(\boldsymbol{\lambda}^{(1)}, \ldots, \boldsymbol{\lambda}^{(k)}\right)$ be a sequence of partitions with at most $N$ parts such that $\sum_{s=1}^{k}\left|\boldsymbol{\lambda}^{(s)}\right|=n$. Denote $n_{s}=\left|\boldsymbol{\lambda}^{(s)}\right|$. Let $\boldsymbol{b}=\left(b_{1}, \ldots, b_{k}\right)$ be a sequence of distinct complex numbers.

Denote by $\Omega_{\boldsymbol{\Lambda}, \overline{\boldsymbol{\lambda}}, \boldsymbol{b}}$ the intersection of the Schubert cells:

$$
\Omega_{\boldsymbol{\Lambda}, \overline{\boldsymbol{\lambda}}, \boldsymbol{b}}=\Omega_{\overline{\boldsymbol{\lambda}}}(\infty) \cap \bigcap_{s=1}^{k} \Omega_{\boldsymbol{\lambda}^{(s)}}\left(b_{s}\right),
$$

where the cell $\Omega_{\bar{\lambda}}(\infty)$ is defined in Section 3.1 Recall that a subspace $X \subset \mathbb{C}_{d}[u]$ is a point of $\Omega_{\bar{\lambda}}(\infty)$ if and only if for every $i=1, \ldots, N$, it contains a monic polynomial of degree $d_{i}$. Similarly, the subspace $X$ is a point of $\Omega_{\boldsymbol{\lambda}^{(s)}}\left(b_{s}\right)$ if and only if for every $i=1, \ldots, N$, it contains a polynomial with a root at $b_{s}$ of order $\lambda_{i}^{(s)}+N-i$.

Given an $N$-dimensional space of polynomials $X \subset \mathbb{C}[u]$, denote by $\mathcal{D}_{X}$ the monic scalar differential operator of order $N$ with kernel $X$. The operator $\mathcal{D}_{X}$ is a Fuchsian differential operator. If $X \in \Omega_{\overline{\boldsymbol{\lambda}}}(\infty)$, then $\mathcal{D}_{X}$ equals the operator $\mathcal{D}_{\boldsymbol{\lambda}}^{\mathcal{O}}$, see (3.5), computed at $X$.

Lemma 4.1. A subspace $X \subset \mathbb{C}_{d}[u]$ is a point of $\Omega_{\boldsymbol{\Lambda}, \overline{\boldsymbol{\lambda}}, \boldsymbol{b}}$ if and only if the singular points of the operator $\mathcal{D}_{X}$ are at $b_{1}, \ldots, b_{k}$ and $\infty$ only, the exponents at $b_{s}, s=$ $1, \ldots, k$, being equal to $\lambda_{N}^{(s)}, \lambda_{N-1}^{(s)}+1, \ldots, \lambda_{1}^{(s)}+N-1$, and the exponents at $\infty$ being equal to $1-N-\lambda_{1}, 2-N-\lambda_{2}, \ldots,-\lambda_{N}$.

Lemma 4.2. Let $\boldsymbol{a}=\left(a_{1}, \ldots, a_{n}\right), \boldsymbol{b}=\left(b_{1}, \ldots, b_{k}\right)$, and $n_{1}, \ldots, n_{k}$ be related as in (2.6). Then $\Omega_{\boldsymbol{\Lambda}, \overline{\boldsymbol{\lambda}}, \boldsymbol{b}} \subset \mathrm{Wr}_{\boldsymbol{\lambda}}^{-1}(\boldsymbol{a})$. In particular, the set $\Omega_{\boldsymbol{\Lambda}, \overline{\boldsymbol{\lambda}}, \boldsymbol{b}}$ is finite.

Let $\mathcal{Q}_{\boldsymbol{\lambda}}$ be the field of fractions of $\mathcal{O}_{\boldsymbol{\lambda}}$ and let $\mathcal{Q}_{\boldsymbol{\Lambda}, \boldsymbol{\lambda}, \boldsymbol{b}} \subset \mathcal{Q}_{\boldsymbol{\lambda}}$ be the subring of elements regular at all points of $\Omega_{\boldsymbol{\Lambda}, \overline{\boldsymbol{\lambda}}, \boldsymbol{b}}$.

Consider the $N \times N$ matrices $M_{1}, \ldots, M_{k}$ with entries in $\mathcal{O}_{\boldsymbol{\lambda}}$,

$$
\left(M_{s}\right)_{i j}=\left.\frac{1}{\left(\lambda_{j}^{(s)}+N-j\right) !}\left(\left(\frac{d}{d u}\right)^{\lambda_{j}^{(s)}+N-j} f_{i}(u)\right)\right|_{u=b_{s}} .
$$

The values of $M_{1}, \ldots, M_{k}$ at any point of $\Omega_{\boldsymbol{\Lambda}, \overline{\boldsymbol{\lambda}}, \boldsymbol{b}}$ are matrices invertible over $\mathbb{C}$. Therefore, the inverse matrices $M_{1}^{-1}, \ldots, M_{k}^{-1}$ exist as matrices with entries in $\mathcal{Q}_{\Lambda, \boldsymbol{\lambda}, \boldsymbol{b}}$.

Introduce the elements $g_{i j s} \in \mathcal{Q}_{\boldsymbol{\Lambda}, \boldsymbol{\lambda}, \boldsymbol{b}}, i=1, \ldots, N, j=0, \ldots, d_{1}, s=1, \ldots, k$, by the rule

$$
\sum_{j=0}^{d_{1}} g_{i j s}\left(u-b_{s}\right)^{j}=\sum_{m=1}^{N}\left(M_{s}^{-1}\right)_{i m} f_{m}(u)
$$

Clearly, $g_{i, \lambda_{j}^{(s)}+N-j, s}=\delta_{i j}$ for all $i, j=1, \ldots, N$, and $s=1, \ldots, k$. 
For each $s=1, \ldots, k$, let $J_{\boldsymbol{\Lambda}, \boldsymbol{\lambda}, \boldsymbol{b}}^{\mathcal{Q}, s}$ be the ideal in $\mathcal{Q}_{\boldsymbol{\Lambda}, \boldsymbol{\lambda}, \boldsymbol{b}}$ generated by the elements $g_{i j s}, i=1, \ldots, N, j=0,1, \ldots, \lambda_{i}^{(s)}+N-i-1$, and $J_{\boldsymbol{\Lambda}, \boldsymbol{\lambda}, \boldsymbol{b}}^{\mathcal{Q}}=\sum_{s=1}^{k} J_{\boldsymbol{\Lambda}, \boldsymbol{\lambda}, \boldsymbol{b}}^{\mathcal{Q}, s}$. Note that the number of generators of the ideal $J_{\boldsymbol{\Lambda}, \boldsymbol{\lambda}, \boldsymbol{b}}^{\mathcal{Q}}$ equals $n$.

The quotient algebra

$$
\mathcal{O}_{\Lambda, \lambda, b}=\mathcal{Q}_{\Lambda, \lambda, b} / J_{\Lambda, \lambda, b}^{\mathcal{Q}}
$$

is the scheme-theoretic intersection of the Schubert cells. We call it the algebra of functions on $\Omega_{\boldsymbol{\Lambda}, \overline{\boldsymbol{\lambda}}, \boldsymbol{b}}$.

Lemma 4.3. The algebra $\mathcal{O}_{\boldsymbol{\Lambda}, \boldsymbol{\lambda}, \boldsymbol{b}}$ is a Frobenius algebra.

Proof. The lemma follows from Lemma 3.9.

It is known from Schubert calculus that

$$
\operatorname{dim} \mathcal{O}_{\boldsymbol{\Lambda}, \boldsymbol{\lambda}, \boldsymbol{b}}=\operatorname{dim}\left(\bigotimes_{s=1}^{k} L_{\boldsymbol{\lambda}^{(s)}}\right)_{\boldsymbol{\lambda}}^{\text {sing }}
$$

for example, see $[\mathrm{Fu}]$.

4.2. Algebra $\Omega_{\boldsymbol{\Lambda}, \boldsymbol{\lambda}, \boldsymbol{b}}$ as quotient of $\mathcal{O}_{\boldsymbol{\lambda}}$. Consider the differential operator

$$
\widetilde{\mathcal{D}}_{\lambda}^{\mathcal{O}}=\operatorname{Wr}\left(f_{1}, \ldots, f_{N}\right) \mathcal{D}_{\lambda}^{\mathcal{O}}=\operatorname{rdet}\left(\begin{array}{cccc}
f_{1}(u) & f_{1}^{\prime}(u) & \ldots & f_{1}^{(N)}(u) \\
f_{2}(u) & f_{2}^{\prime}(u) & \ldots & f_{2}^{(N)}(u) \\
\ldots & \ldots & \ldots & \ldots \\
1 & \partial & \ldots & \partial^{N}
\end{array}\right) .
$$

It is a differential operator in the variable $u$ whose coefficients are polynomials in $u$ with coefficients in $\mathcal{O}_{\boldsymbol{\lambda}}$,

$$
\widetilde{\mathcal{D}}_{\boldsymbol{\lambda}}^{\mathcal{O}}=\sum_{i=0}^{N} G_{i}(u) \partial^{N-i} .
$$

Clearly, if $n-i<0$, then $G_{i}(u)=0$; otherwise $\operatorname{deg} G_{i}=n-i$ and

$$
G_{i}(u)=\operatorname{Wr}\left(f_{1}(u), \ldots, f_{N}(u)\right) F_{i}(u),
$$

where $i=0, \ldots, N$, and $F_{0}(u)=1$.

Introduce the elements $G_{i j s} \in \mathcal{O}_{\boldsymbol{\lambda}}, i=0, \ldots, N, j=0,1, \ldots, n-i, s=1, \ldots, k$, by the rule

$$
G_{i}(u)=\sum_{j=0}^{n-i} G_{i j s}\left(u-b_{s}\right)^{j} .
$$

We set $G_{i j s}=0$ if $j<0$ or $j>n-i$.

Define the indicial polynomial $\chi_{s}^{\mathcal{O}}(\alpha)$ at $b_{s}$ by the formula

$$
\chi_{s}^{\mathcal{O}}(\alpha)=\sum_{i=0}^{N} G_{i, n_{s}-i, s} \prod_{j=0}^{N-i-1}(\alpha-j) .
$$

It is a polynomial of degree $N$ in the variable $\alpha$ with coefficients in $\mathcal{O}_{\boldsymbol{\lambda}}$.

Lemma 4.4. For a complex number $r$, the element $\chi_{s}^{\mathcal{O}}(r)$ is invertible in $\mathcal{Q}_{\boldsymbol{\Lambda}, \boldsymbol{\lambda}, \boldsymbol{b}}$ provided $r \neq \lambda_{j}^{(s)}+N-j$ for all $j=1, \ldots, N$.

Proof. An element of $\mathcal{Q}_{\boldsymbol{\Lambda}, \boldsymbol{\lambda}, \boldsymbol{b}}$ is invertible if and only if its value at any point of $\Omega_{\boldsymbol{\Lambda}, \overline{\boldsymbol{\lambda}}, \boldsymbol{b}}$ is nonzero. Now the claim follows from Lemmas 4.1 and 4.2 
For each $s=1, \ldots, k$, let $I_{\boldsymbol{\Lambda}, \boldsymbol{\lambda}, \boldsymbol{b}}^{\mathcal{Q}, \boldsymbol{s}}$ be the ideal in $\mathcal{Q}_{\boldsymbol{\Lambda}, \boldsymbol{\lambda}, \boldsymbol{b}}$ generated by the elements $G_{i j s}, i=0, \ldots, N, j=0,1, \ldots, n_{s}-i-1$, and the coefficients of the polynomials

$$
\chi_{s}^{\mathcal{O}}(\alpha)-\prod_{\substack{r=1 \\ r \neq s}}^{k}\left(b_{s}-b_{r}\right)^{n_{r}} \prod_{l=1}^{N}\left(\alpha-\lambda_{l}^{(s)}-N+l\right), \quad s=1, \ldots, k .
$$

Denote $I_{\boldsymbol{\Lambda}, \boldsymbol{\lambda}, \boldsymbol{b}}^{\mathcal{Q}}=\sum_{s=1}^{k} I_{\boldsymbol{\Lambda}, \boldsymbol{\lambda}, \boldsymbol{b}}^{\mathcal{Q}, s}$.

Lemma 4.5. For any $s=1, \ldots, k$, the ideals $I_{\boldsymbol{\Lambda}, \boldsymbol{\lambda}, \boldsymbol{b}}^{\mathcal{Q}, s}$ and $J_{\boldsymbol{\Lambda}, \boldsymbol{\lambda}, \boldsymbol{b}}^{\mathcal{Q}, s}$ coincide.

Proof. Consider the differential operator $\widetilde{\mathcal{D}}_{\boldsymbol{\lambda}}^{\mathcal{O}}$, given by (4.5). We have

$$
\widetilde{\mathcal{D}}_{\boldsymbol{\lambda}}^{\mathcal{O}}=\sum_{i=0}^{N} \sum_{j=0}^{n-i} G_{i j s}\left(u-b_{s}\right)^{j} \partial^{N-i},
$$

see (3.6), (4.7), and

$$
\widetilde{\mathcal{D}}_{\lambda}^{\mathcal{O}}=\operatorname{det} M_{s} \cdot \operatorname{rdet}\left(\begin{array}{cccc}
g_{1 s}(u) & g_{1 s}^{\prime}(u) & \ldots & g_{1 s}^{(N)}(u) \\
g_{2 s}(u) & g_{2 s}^{\prime}(u) & \ldots & g_{2 s}^{(N)}(u) \\
\ldots & \ldots & \ldots & \ldots \\
1 & \partial & \ldots & \partial^{N}
\end{array}\right),
$$

where $g_{i s}(u)=\sum_{j=0}^{d_{1}} g_{i j s}\left(u-b_{s}\right)^{j}$; see (3.5), (4.2). Formulae (4.9) and (4.10) imply that $I_{\boldsymbol{\Lambda}, \boldsymbol{\lambda}, \boldsymbol{b}}^{\mathcal{Q}, s} \subset J_{\boldsymbol{\Lambda}, \boldsymbol{\lambda}, \boldsymbol{b}}^{\mathcal{Q}, s}$.

To get the opposite inclusion, $J_{\boldsymbol{\Lambda}, \boldsymbol{\lambda}, \boldsymbol{b}}^{\mathcal{Q}, s} \subset I_{\boldsymbol{\Lambda}, \boldsymbol{\lambda}, \boldsymbol{b}}^{\mathcal{Q}, s}$, write $\widetilde{\mathcal{D}}_{\boldsymbol{\lambda}}^{\mathcal{O}} g_{i s}(u)=\sum_{j=0}^{n-N} q_{i j s}(u-$ $\left.b_{s}\right)^{j}$. Then

$$
q_{i, n_{s}-N+r, s}=\sum_{j=0}^{d_{1}} \sum_{l=0}^{N} g_{i j s} G_{l, n_{s}+r-j-l, s} \prod_{m=0}^{N-l-1}(j-m),
$$

where it is assumed that the elements $g_{i j s}$ and $G_{l, n_{s}+r-j-l, s}$ equal zero if their subscripts are out of range of the definition. Observe that the terms with $j=r$ in the right-hand side of (4.11) sum to $\chi_{s}^{\mathcal{O}}(r) g_{\text {irs }}$, and the terms with $j>r$ belong to the ideal $I_{\boldsymbol{\Lambda}, \boldsymbol{\lambda}, \boldsymbol{b}}^{\mathcal{Q}, s}$.

We have $\widetilde{\mathcal{D}}_{\boldsymbol{\lambda}}^{\mathcal{O}} g_{i s}(u)=0$, so that $q_{i, n_{s}-N+r, s}=0$. Using Lemma 4.4 and taking into account that $g_{i, \lambda_{l}^{(s)}+N-l, s}=0$ for $l>r$, we can show recursively that the elements $g_{\text {irs }}$ with $r<\lambda_{i}^{(s)}+N-i$ belong to $I_{\boldsymbol{\Lambda}, \boldsymbol{\lambda}, \boldsymbol{b}}^{\mathcal{Q}, s}$, starting with $r=0$ and then increasing the second index $r$. Therefore, $J_{\boldsymbol{\Lambda}, \boldsymbol{\lambda}, \boldsymbol{b}}^{\mathcal{Q}, s} \subset I_{\boldsymbol{\Lambda}, \boldsymbol{\lambda}, \boldsymbol{b}}^{\mathcal{Q}, s}$.

Let $I_{\boldsymbol{\Lambda}, \boldsymbol{\lambda}, \boldsymbol{b}}^{\mathcal{O}}$ be the ideal in $\mathcal{O}_{\boldsymbol{\lambda}}$ generated by the elements $G_{i j s}, i=0, \ldots, N$, $s=1, \ldots, k, j=0,1, \ldots, n_{s}-i-1$, and the coefficients of polynomials (4.8).

Proposition 4.6. The algebra $\mathcal{O}_{\boldsymbol{\Lambda}, \boldsymbol{\lambda}, \boldsymbol{b}}$ is isomorphic to the quotient algebra $\mathcal{O}_{\boldsymbol{\lambda}} / I_{\Lambda, \lambda, b}^{\mathcal{O}}$.

Proof. By Lemma 4.5, the ideals $I_{\boldsymbol{\Lambda}, \boldsymbol{\lambda}, \boldsymbol{b}}^{\mathcal{Q}}$ and $J_{\boldsymbol{\Lambda}, \boldsymbol{\lambda}, \boldsymbol{b}}^{\mathcal{Q}}$ coincide, so the algebra $\mathcal{O}_{\boldsymbol{\Lambda}, \boldsymbol{\lambda}, \boldsymbol{b}}$ is isomorphic to the quotient algebra $\mathcal{Q}_{\boldsymbol{\Lambda}, \boldsymbol{\lambda}, \boldsymbol{b}} / I_{\boldsymbol{\Lambda}, \boldsymbol{\lambda}, \boldsymbol{b}}^{\mathcal{O}}$. By Lemma 4.1, the algebraic set defined by the ideal $I_{\boldsymbol{\Lambda}, \boldsymbol{\lambda}, \boldsymbol{b}}^{\mathcal{O}}$ equals $\Omega_{\boldsymbol{\Lambda}, \overline{\boldsymbol{\lambda}}, \boldsymbol{b}}$. The set $\Omega_{\boldsymbol{\Lambda}, \overline{\boldsymbol{\lambda}}, \boldsymbol{b}}$ is finite by Lemma4.2. Therefore, the quotient algebras $\mathcal{Q}_{\boldsymbol{\Lambda}, \boldsymbol{\lambda}, \boldsymbol{b}} / I_{\boldsymbol{\Lambda}, \boldsymbol{\lambda}, \boldsymbol{b}}^{\mathcal{Q}}$ and $\mathcal{O}_{\boldsymbol{\lambda}} / I_{\boldsymbol{\Lambda}, \boldsymbol{\lambda}, \boldsymbol{b}}^{\mathcal{O}}$ are isomorphic. 
4.3. Algebra $\mathcal{O}_{\boldsymbol{\Lambda}, \boldsymbol{\lambda}, \boldsymbol{b}}$ as quotient of $\mathcal{O}_{\boldsymbol{\lambda}, \boldsymbol{a}}$. Recall that $\mathcal{O}_{\boldsymbol{\lambda}, \boldsymbol{a}}=\mathcal{O}_{\boldsymbol{\lambda}} / I_{\boldsymbol{\lambda}, \boldsymbol{a}}^{\mathcal{O}}$ is the algebra of functions on $\mathrm{Wr}_{\boldsymbol{\lambda}}^{-1}(\boldsymbol{a})$; see (3.9). For an element $F \in \mathcal{O}_{\boldsymbol{\lambda}}$, we denote by $\bar{F}$ the projection of $F$ to the quotient algebra $\mathcal{O}_{\boldsymbol{\lambda}, \boldsymbol{a}}$.

Lemma 4.7. The elements $\bar{G}_{i j s}, i=1, \ldots, N, s=1, \ldots, k, j=0,1, \ldots, n_{s}-i-1$, are nilpotent.

Proof. The values of these elements on every element $X \in \mathrm{Wr}^{-1}(\boldsymbol{a})$ are clearly zero. The lemma follows.

Define the indicial polynomial $\bar{\chi}_{s}^{\mathcal{O}}(\alpha)$ at $b_{s}$ by the formula

$$
\bar{\chi}_{s}^{\mathcal{O}}(\alpha)=\sum_{i=0}^{N} \bar{G}_{i, n_{s}-i, s} \prod_{j=0}^{N-i-1}(\alpha-j) .
$$

Let $\bar{I}_{\boldsymbol{\Lambda}, \boldsymbol{\lambda}, \boldsymbol{b}}^{\mathcal{O}}$ be the ideal in $\mathcal{O}_{\boldsymbol{\lambda}, \boldsymbol{a}}$ generated by the elements $\bar{G}_{i j s}, i=1, \ldots, N$, $s=1, \ldots, k, j=0,1, \ldots, n_{s}-i-1$, and the coefficients of the polynomials

$$
\bar{\chi}_{s}^{\mathcal{O}}(\alpha)-\prod_{\substack{r=1 \\ r \neq s}}^{k}\left(b_{s}-b_{r}\right)^{n_{r}} \prod_{l=1}^{N}\left(\alpha-\lambda_{l}^{(s)}-N+l\right), \quad s=1, \ldots, k .
$$

Proposition 4.8. The algebra $\mathcal{O}_{\boldsymbol{\Lambda}, \boldsymbol{\lambda}, \boldsymbol{b}}$ is isomorphic to the quotient algebra $\mathcal{O}_{\boldsymbol{\lambda}, \boldsymbol{a}} / \bar{I}_{\Lambda, \boldsymbol{\lambda}, \boldsymbol{b}}^{\mathcal{O}}$

Proof. The elements $G_{0 j s}, j=0, \ldots, n_{s}-1, s=1, \ldots, k$, generate the ideal $I_{\boldsymbol{\lambda}, \boldsymbol{a}}^{\mathcal{O}}$ in $\mathcal{O}_{\boldsymbol{\lambda}}$. Moreover, the projection of the ideal $I_{\boldsymbol{\Lambda}, \boldsymbol{\lambda}, \boldsymbol{b}}^{\mathcal{O}} \subset \mathcal{O}_{\boldsymbol{\lambda}}$ to $\mathcal{O}_{\boldsymbol{\lambda}, \boldsymbol{a}}$ equals $\bar{I}_{\boldsymbol{\Lambda}, \boldsymbol{\lambda}, \boldsymbol{b}}^{\mathcal{O}}$. Hence, the proposition follows from Proposition 4.6 .

Example. Let $N=2, n=3, d>4, \boldsymbol{\lambda}=(2,1), \boldsymbol{a}=(0,0,0)$. Then $k=1, \boldsymbol{b}=\left(b_{1}\right)$ with $b_{1}=0$, and $n_{1}=3$, and we have

$$
\begin{gathered}
f_{1}(u)=u^{3}+f_{11} u^{2}+f_{13}, \quad f_{2}(u)=u+f_{21}, \\
\operatorname{Wr}\left(f_{1}(u), f_{2}(u)\right)=-2 u^{3}-\left(f_{11}+3 f_{21}\right) u^{2}-2 f_{11} f_{21} u+f_{13}, \\
\mathcal{O}_{\boldsymbol{\lambda}, \boldsymbol{a}}=\mathbb{C}\left[f_{11}, f_{13}, f_{21}\right] /\left\langle\left(f_{11}+3 f_{21}\right), 2 f_{11} f_{21}, f_{13}\right\rangle,
\end{gathered}
$$

so the algebra $\mathcal{O}_{\boldsymbol{\lambda}, \boldsymbol{a}}$ equals $\mathbb{C} 1+\mathbb{C} \bar{f}_{11}$ with $\bar{f}_{11}^{2}=0$.

If $\boldsymbol{\Lambda}=\left(\boldsymbol{\lambda}^{(1)}\right)$ with $\boldsymbol{\lambda}^{(1)}=(2,1)$, then the ideal $\bar{I}_{\boldsymbol{\Lambda}, \boldsymbol{\lambda}, \boldsymbol{b}}^{\mathcal{O}}$ is generated by the element $\bar{f}_{11}$ and $\operatorname{dim} \mathcal{O}_{\boldsymbol{\lambda}, \boldsymbol{a}} / \bar{I}_{\boldsymbol{\Lambda}, \boldsymbol{\lambda}, \boldsymbol{b}}^{\mathcal{O}}=1$.

If $\boldsymbol{\Lambda}=\left(\boldsymbol{\lambda}^{(1)}\right)$ with $\boldsymbol{\lambda}^{(1)}=(3,0)$, then the ideal $\bar{I}_{\boldsymbol{\Lambda}, \boldsymbol{\lambda}, \boldsymbol{b}}^{\mathcal{O}}$ is generated by the elements 3 and $\bar{f}_{11}$, and $\operatorname{dim} \mathcal{O}_{\boldsymbol{\lambda}, \boldsymbol{a}} / \bar{I}_{\boldsymbol{\Lambda}, \boldsymbol{\lambda}, \boldsymbol{b}}^{\mathcal{O}}=0$.

Recall that the ideal $\operatorname{Ann}\left(\bar{I}_{\boldsymbol{\Lambda}, \boldsymbol{\lambda}, \boldsymbol{b}}^{\mathcal{O}}\right) \subset \mathcal{O}_{\boldsymbol{\lambda}, \boldsymbol{a}}$ is naturally an $\mathcal{O}_{\boldsymbol{\Lambda}, \boldsymbol{\lambda}, \boldsymbol{b}}$-module.

Corollary 4.9. The $\mathcal{O}_{\boldsymbol{\Lambda}, \boldsymbol{\lambda}, \boldsymbol{b}}$-module $\operatorname{Ann}\left(\bar{I}_{\boldsymbol{\Lambda}, \boldsymbol{\lambda}, \boldsymbol{b}}^{\mathcal{O}}\right)$ is isomorphic to the coregular representation of $\mathcal{O}_{\boldsymbol{\Lambda}, \boldsymbol{\lambda}, \boldsymbol{b}}$ on the dual space $\left(\mathcal{O}_{\boldsymbol{\Lambda}, \boldsymbol{\lambda}, \boldsymbol{b}}\right)^{*}$.

Proof. The statement follows from Lemmas 4.3 and 3.8 


\section{THREE ISOMORPHISMS}

5.1. Case of generic $\boldsymbol{a}$. Fix natural numbers $n$ and $d \geqslant N$, and a partition $\boldsymbol{\lambda}=\left(\lambda_{1}, \ldots, \lambda_{N}\right)$ such that $|\boldsymbol{\lambda}|=n$ and $\lambda_{1} \leqslant d-N$. Define the partition $\overline{\boldsymbol{\lambda}}$ by formula (3.1).

Recall that given an $N$-dimensional space of polynomials $X \subset \mathbb{C}[u]$, we denote by $\mathcal{D}_{X}$ the monic scalar differential operator of order $N$ with kernel $X$.

Let $M$ be $\mathfrak{a l}_{N}[t]$-module $M$ and $v$ an eigenvector of the Bethe algebra $\mathcal{B} \subset$ $U\left(\mathfrak{g l}_{N}[t]\right)$ acting on $M$. Then for any coefficient $B_{i}(u)$ of the universal differential operator $\mathcal{D}^{\mathcal{B}}$ we have $B_{i}(u) v=h_{i}(u) v$, where $h_{i}(u)$ is a scalar series. We call the scalar differential operator

$$
\mathcal{D}_{v}^{\mathcal{B}}=\partial^{N}+\sum_{i=1}^{N} h_{i}(u) \partial^{N-i}
$$

the differential operator associated with $v$.

Lemma 5.1. There exist a Zariski open $S_{n}$-invariant subset $\Theta$ of $\mathbb{C}^{n}$ and a Zariski open subset $\Xi$ of $\Omega_{\bar{\lambda}}(\infty)$ with the following properties.

(i) For any $\left(b_{1}, \ldots, b_{n}\right) \in \Theta$, all numbers $b_{1}, \ldots, b_{n}$ are distinct, and there exists a basis of $\left(\bigotimes_{s=1}^{n} V\left(b_{s}\right)\right)_{\lambda}^{\text {sing }}$ such that every basis vector $v$ is an eigenvector of the Bethe algebra and $\mathcal{D}_{v}^{\mathcal{B}}=\mathcal{D}_{X}$ for some $X \in \Xi$. Moreover, different basis vectors correspond to different points of $\Xi$.

(ii) For any $X \in \Xi$, if $b_{1}, \ldots, b_{n}$ are all roots of the Wronskian $\mathrm{Wr}_{X}$, then $\left(b_{1}, \ldots, b_{n}\right) \in \Theta$, and there exists a unique up to proportionality vector $v \in\left(\bigotimes_{s=1}^{n} V\left(b_{s}\right)\right)_{\lambda}^{\text {sing }}$ such that $v$ is an eigenvector of the Bethe algebra and $\mathcal{D}_{v}^{\mathcal{B}}=\mathcal{D}_{X}$.

Proof. The basis in part (i) is constructed by the Bethe ansatz method; see MV2. The equality $\mathcal{D}_{v}^{\mathcal{B}}=\mathcal{D}_{X}$ is proved in [MTV1. The existence of an eigenvector $v$ in part (ii) for generic $X \subset \Omega_{\bar{\lambda}}$ is proved in [MV1].

For generic $\boldsymbol{a}=\left(a_{1}, \ldots, a_{n}\right)$, the roots $\left(b_{1}, \ldots, b_{n}\right)$ of the polynomial $\sum_{i=0}^{n} a_{i} x^{i}$ form a point in $\Theta$. Therefore, Lemma [5.1 in particular, asserts that for generic values of $\boldsymbol{a} \in \mathbb{C}^{n}$, the algebras $\mathcal{B}_{\boldsymbol{\lambda}, \boldsymbol{a}}$ and $\mathcal{O}_{\boldsymbol{\lambda}, \boldsymbol{a}}$ are both isomorphic to the direct sum of $\operatorname{dim}\left(V^{\otimes n}\right)_{\lambda}^{\operatorname{sing}}$ copies of $\mathbb{C}$.

The following corollary recovers a well-known fact.

Corollary 5.2. The degree of the Wronski map equals $\operatorname{dim}\left(V^{\otimes n}\right)_{\boldsymbol{\lambda}}^{\text {sing }}$.

5.2. Isomorphism of algebras $\mathcal{O}_{\boldsymbol{\lambda}}$ and $\mathcal{B}_{\boldsymbol{\lambda}}$. In this section we show that the Bethe algebra $\mathcal{B}_{\boldsymbol{\lambda}}$, associated with the space $\mathcal{M}_{\boldsymbol{\lambda}}=\left(\mathcal{V}^{S}\right)_{\lambda}^{\text {sing }}$, see (2.12), is isomorphic to the algebra $\mathcal{O}_{\boldsymbol{\lambda}}$ of regular functions of the Schubert cell $\Omega_{\bar{\lambda}}(\infty)$, and that under this isomorphism the $\mathcal{B}_{\boldsymbol{\lambda}}$-module $\mathcal{M}_{\boldsymbol{\lambda}}$ is isomorphic to the regular representation of $\mathcal{O}_{\lambda}$.

Consider the map

$$
\tau_{\boldsymbol{\lambda}}: \mathcal{O}_{\boldsymbol{\lambda}} \rightarrow \mathcal{B}_{\boldsymbol{\lambda}}, \quad F_{i j} \mapsto \hat{B}_{i j},
$$

where the elements $F_{i j} \in \mathcal{O}_{\boldsymbol{\lambda}}$ are defined by (3.7) and $\hat{B}_{i j} \in \mathcal{B}_{\boldsymbol{\lambda}}$ are the images of the elements $B_{i j} \in \mathcal{B}$, defined by (2.9).

Theorem 5.3. The map $\tau_{\boldsymbol{\lambda}}$ is a well-defined isomorphism of graded algebras. 
Proof. Let a polynomial $R\left(F_{i j}\right)$ in the generators $F_{i j}$ be equal to zero in $\mathcal{O}_{\boldsymbol{\lambda}}$. Let us prove that the corresponding polynomial $R\left(\hat{B}_{i j}\right)$ is equal to zero in the $\mathcal{B}_{\boldsymbol{\lambda}}$. Indeed, $R\left(\hat{B}_{i j}\right)$ is a polynomial in $z_{1}, \ldots, z_{n}$ with values in $\operatorname{End}\left(\left(V^{\otimes n}\right)_{\lambda}^{\operatorname{sing}}\right)$. Let $\Theta$ be the set, introduced in Lemma [5.1, and let $\left(b_{1}, \ldots, b_{n}\right) \in \Theta$. Then by part (i) of Lemma 5.1, the value of the polynomial $R\left(\hat{B}_{i j}\right)$ at $z_{1}=b_{1}, \ldots, z_{n}=b_{n}$ equals zero. Hence, the polynomial $R\left(\hat{B}_{i j}\right)$ equals zero identically and the map $\tau_{\boldsymbol{\lambda}}$ is well defined.

By Lemmas 3.2 and 2.16 the elements $F_{i j}$ and $\hat{B}_{i j}$ are of the same degree. Therefore, the map $\tau_{\boldsymbol{\lambda}}$ is graded.

Let a polynomial $R\left(F_{i j}\right)$ in the generators $F_{i j}$ be a nonzero element of $\mathcal{O}_{\boldsymbol{\lambda}}$. Then the value of $R\left(F_{i j}\right)$ at a generic point $X \in \Omega_{\bar{\lambda}}(\infty)$ is not equal to zero. Then by part (ii) of Lemma 5.1 the polynomial $R\left(\hat{B}_{i j}\right)$ is not identically equal to zero. Therefore, the map $\tau_{\boldsymbol{\lambda}}$ is injective.

Since the elements $\hat{B}_{i j}$ generate the algebra $\mathcal{B}_{\boldsymbol{\lambda}}$, the map $\tau_{\boldsymbol{\lambda}}$ is surjective.

The algebra $\mathbb{C}\left[z_{1}, \ldots, z_{n}\right]^{S}$ is embedded into the algebra $\mathcal{B}_{\boldsymbol{\lambda}}$ as the subalgebra of operators of multiplication by symmetric polynomials; see Lemma 2.10 and formula (2.11). The algebra $\mathbb{C}\left[z_{1}, \ldots, z_{n}\right]^{S}$ is embedded into the algebra $\mathcal{O}_{\boldsymbol{\lambda}}$, the elementary symmetric polynomials $\sigma_{1}(\boldsymbol{z}), \ldots, \sigma_{n}(\boldsymbol{z})$ being mapped to the elements $\Sigma_{1}, \ldots, \Sigma_{n}$, defined by (3.4). These embeddings give the algebras $\mathcal{B}_{\boldsymbol{\lambda}}$ and $\mathcal{O}_{\boldsymbol{\lambda}}$ the structure of $\mathbb{C}\left[z_{1}, \ldots, z_{n}\right]^{S}$-modules.

Lemma 5.4. We have $\tau_{\boldsymbol{\lambda}}\left(\Sigma_{i}\right)=\sigma_{i}(\boldsymbol{z})$ for all $i=1, \ldots, n$. In particular, the map $\tau_{\boldsymbol{\lambda}}: \mathcal{O}_{\boldsymbol{\lambda}} \rightarrow \mathcal{B}_{\boldsymbol{\lambda}}$ is an isomorphism of $\mathbb{C}\left[z_{1}, \ldots, z_{n}\right]^{S}$-modules.

Proof. The lemma follows from the fact that

$$
F_{1}(u)=-\frac{\mathrm{Wr}^{\prime}\left(f_{1}(u), \ldots, f_{N}(u)\right)}{\operatorname{Wr}\left(f_{1}(u), \ldots, f_{N}(u)\right)},
$$

where ${ }^{\prime}$ denotes the derivative with respect to $u$, and formula (2.10).

Given a vector $v \in \mathcal{M}_{\boldsymbol{\lambda}}$, consider a linear map

$$
\mu_{v}: \mathcal{O}_{\boldsymbol{\lambda}} \rightarrow \mathcal{M}_{\boldsymbol{\lambda}}, \quad F \mapsto \tau_{\boldsymbol{\lambda}}(F) v .
$$

Lemma 5.5. If $v \in \mathcal{M}_{\boldsymbol{\lambda}}$ is nonzero, then the map $\mu_{v}$ is injective.

Proof. The algebra $\mathcal{O}_{\boldsymbol{\lambda}}$ is a free polynomial algebra containing the subalgebra $\mathbb{C}\left[z_{1}, \ldots, z_{n}\right]^{S}$. By Lemma 3.11, the quotient algebra $\mathcal{O}_{\boldsymbol{\lambda}} / \mathbb{C}\left[z_{1}, \ldots, z_{n}\right]^{S}$ is finitedimensional. The kernel of $\mu_{v}$ is an ideal in $\mathcal{B}_{\boldsymbol{\lambda}}$ which has zero intersection with $\mathbb{C}\left[z_{1}, \ldots, z_{n}\right]^{S}$ and, therefore, is the zero ideal.

By Lemmas 2.9 and 2.12 , the space $\mathcal{M}_{\boldsymbol{\lambda}}$ is a free graded $\mathbb{C}\left[z_{1}, \ldots, z_{n}\right]^{S}$-module. By Lemma 2.13, the generators of this module can be identified with a basis in $\left(W_{n}\right)_{\boldsymbol{\lambda}}^{s i n g}$. Therefore, the graded character of $\mathcal{M}_{\boldsymbol{\lambda}}$ is given by the formula

$$
\operatorname{ch}\left(\mathcal{M}_{\boldsymbol{\lambda}}\right)=\frac{\operatorname{ch}\left(\left(W_{n}\right)_{\boldsymbol{\lambda}}^{\text {sing }}\right)}{(q)_{n}} .
$$

This equality and part (iv) of Lemma 2.2 imply that the degree of any vector in $\mathcal{M}_{\boldsymbol{\lambda}}$ is at least $\sum_{i=1}^{N}(i-1) \lambda_{i}$ and the homogeneous component of $\mathcal{M}_{\boldsymbol{\lambda}}$ of degree $\sum_{i=1}^{N}(i-1) \lambda_{i}$ is one-dimensional.

Fix a nonzero vector $v \in \mathcal{M}_{\boldsymbol{\lambda}}$ of degree $\sum_{i=1}^{N}(i-1) \lambda_{i}$. Denote the map $\mu_{v}$ by $\mu_{\lambda}$. 
Theorem 5.6. The map $\mu_{\boldsymbol{\lambda}}: \mathcal{O}_{\boldsymbol{\lambda}} \rightarrow \mathcal{M}_{\boldsymbol{\lambda}}$ is an isomorphism of degree $\sum_{i=1}^{N}(i-1) \lambda_{i}$ of graded vector spaces. For any $F, G \in \mathcal{O}_{\boldsymbol{\lambda}}$, we have

$$
\mu_{\boldsymbol{\lambda}}(F G)=\tau_{\boldsymbol{\lambda}}(F) \mu_{\boldsymbol{\lambda}}(G) .
$$

In other words, the maps $\tau_{\boldsymbol{\lambda}}$ and $\mu_{\boldsymbol{\lambda}}$ give an isomorphism of the regular representation of $\mathcal{O}_{\boldsymbol{\lambda}}$ and the $\mathcal{B}_{\boldsymbol{\lambda}}$-module $\mathcal{M}_{\boldsymbol{\lambda}}$.

Proof. The map $\mu_{\boldsymbol{\lambda}}$ is injective by Lemma [5.5. The map $\mu_{\boldsymbol{\lambda}}$ shifts the degree by $\sum_{i=1}^{N}(i-1) \lambda_{i}$. Lemma 3.1] formula (5.3), and part (iv) of Lemma 2.2 imply that $\operatorname{ch}\left(\mu_{\boldsymbol{\lambda}}\left(\mathcal{O}_{\boldsymbol{\lambda}}\right)\right)=\operatorname{ch}\left(\mathcal{M}_{\boldsymbol{\lambda}}\right)$. Hence, the map $\mu_{\boldsymbol{\lambda}}$ is surjective. Formula (5.4) follows from Theorem 5.3 .

5.3. Isomorphism of algebras $\mathcal{O}_{\boldsymbol{\lambda}, \boldsymbol{a}}$ and $\mathcal{B}_{\boldsymbol{\lambda}, \boldsymbol{a}}$. Let $\boldsymbol{a}=\left(a_{1}, \ldots, a_{n}\right)$ be a sequence of complex numbers. Let distinct complex numbers $b_{1}, \ldots, b_{k}$ and integers $n_{1}, \ldots, n_{k}$ be given by (2.6).

In this section we show that the Bethe algebra $\mathcal{B}_{\boldsymbol{\lambda}, \boldsymbol{a}}$, associated with the space $\mathcal{M}_{\boldsymbol{\lambda}, \boldsymbol{a}}=\left(\bigotimes_{s=1}^{k} W_{n_{s}}\left(b_{s}\right)\right)_{\boldsymbol{\lambda}}^{s i n g}$, see (2.12) , is isomorphic to the algebra $\mathcal{O}_{\boldsymbol{\lambda}, \boldsymbol{a}}$ of functions on the preimage $\mathrm{Wr}_{\boldsymbol{\lambda}}^{-1}(\boldsymbol{a})$. We also show that under this isomorphism the $\mathcal{B}_{\boldsymbol{\lambda}, \boldsymbol{a}}$-module $\mathcal{M}_{\boldsymbol{\lambda}, \boldsymbol{a}}$ is isomorphic to the regular representation of $\mathcal{O}_{\boldsymbol{\lambda}, \boldsymbol{a}}$.

Let $I_{\boldsymbol{\lambda}, \boldsymbol{a}}^{\mathcal{B}} \subset \mathcal{B}_{\boldsymbol{\lambda}}$ be the ideal generated by the elements $\sigma_{i}(\boldsymbol{z})-a_{i}, i=1, \ldots, n$. Consider the subspace $I_{\boldsymbol{\lambda}, \boldsymbol{a}}^{\mathcal{M}}=I_{\boldsymbol{\lambda}, \boldsymbol{a}}^{\mathcal{B}} \mathcal{M}_{\boldsymbol{\lambda}}=I_{\boldsymbol{a}}^{\mathcal{V}} \cap \mathcal{M}_{\boldsymbol{\lambda}}$, where $I_{\boldsymbol{a}}^{\mathcal{V}}$ is given by (2.5). Recall the ideal $I_{\boldsymbol{\lambda}, \boldsymbol{a}}^{\mathcal{O}}$ defined in Section 3.4 .

Lemma 5.7. We have

$$
\tau_{\boldsymbol{\lambda}}\left(I_{\boldsymbol{\lambda}, \boldsymbol{a}}^{\mathcal{O}}\right)=I_{\boldsymbol{\lambda}, \boldsymbol{a}}^{\mathcal{B}}, \quad \mu_{\boldsymbol{\lambda}}\left(I_{\boldsymbol{\lambda}, \boldsymbol{a}}^{\mathcal{O}}\right)=I_{\boldsymbol{\lambda}, \boldsymbol{a}}^{\mathcal{M}}, \quad \mathcal{B}_{\boldsymbol{\lambda}, \boldsymbol{a}}=\mathcal{B}_{\boldsymbol{\lambda}} / I_{\boldsymbol{\lambda}, \boldsymbol{a}}^{\mathcal{B}}, \quad \mathcal{M}_{\boldsymbol{\lambda}, \boldsymbol{a}}=\mathcal{M}_{\boldsymbol{\lambda}} / I_{\boldsymbol{\lambda}, \boldsymbol{a}}^{\mathcal{M}} .
$$

Proof. The lemma follows from Theorems 5.3, 5.6 and Lemmas 5.4, 2.13

By Lemma 5.7 the maps $\tau_{\boldsymbol{\lambda}}$ and $\mu_{\boldsymbol{\lambda}}$ induce the maps

$$
\tau_{\boldsymbol{\lambda}, \boldsymbol{a}}: \mathcal{O}_{\boldsymbol{\lambda}, \boldsymbol{a}} \rightarrow \mathcal{B}_{\boldsymbol{\lambda}, \boldsymbol{a}}, \quad \mu_{\boldsymbol{\lambda}, \boldsymbol{a}}: \mathcal{O}_{\boldsymbol{\lambda}, \boldsymbol{a}} \rightarrow \mathcal{M}_{\boldsymbol{\lambda}, \boldsymbol{a}} .
$$

Theorem 5.8. The map $\tau_{\boldsymbol{\lambda}, \boldsymbol{a}}$ is an isomorphism of algebras. The map $\mu_{\boldsymbol{\lambda}, \boldsymbol{a}}$ is an isomorphism of vector spaces. For any $F, G \in \mathcal{O}_{\boldsymbol{\lambda}, \boldsymbol{a}}$, we have

$$
\mu_{\boldsymbol{\lambda}, \boldsymbol{a}}(F G)=\tau_{\boldsymbol{\lambda}, \boldsymbol{a}}(F) \mu_{\boldsymbol{\lambda}, \boldsymbol{a}}(G) .
$$

In other words, the maps $\tau_{\boldsymbol{\lambda}, \boldsymbol{a}}$ and $\mu_{\boldsymbol{\lambda}, \boldsymbol{a}}$ give an isomorphism of the regular representation of $\mathcal{O}_{\boldsymbol{\lambda}, \boldsymbol{a}}$ and the $\mathcal{B}_{\boldsymbol{\lambda}, \boldsymbol{a}}$-module $\mathcal{M}_{\boldsymbol{\lambda}, \boldsymbol{a}}$.

Proof. The theorem follows from Theorems 5.3, 5.6 and Lemma 5.7.

Remark. By Lemma 3.11, the algebra $\mathcal{O}_{\boldsymbol{\lambda}, \boldsymbol{a}}$ is Frobenius. Therefore, its regular and coregular representations are isomorphic.

5.4. Isomorphism of algebras $\mathcal{O}_{\boldsymbol{\Lambda}, \boldsymbol{\lambda}, \boldsymbol{b}}$ and $\mathcal{B}_{\boldsymbol{\Lambda}, \boldsymbol{\lambda}, \boldsymbol{b}}$. Let $\boldsymbol{\Lambda}=\left(\boldsymbol{\lambda}^{(1)}, \ldots, \boldsymbol{\lambda}^{(k)}\right)$ be a sequence of partitions with at most $N$ parts such that $\left|\boldsymbol{\lambda}^{(s)}\right|=n_{s}$ for all $s=1, \ldots, k$.

In this section we show that the Bethe algebra $\mathcal{B}_{\boldsymbol{\Lambda}, \boldsymbol{\lambda}, \boldsymbol{b}}$, associated with the space $\mathcal{M}_{\boldsymbol{\Lambda}, \boldsymbol{\lambda}, \boldsymbol{b}}=\left(\bigotimes_{s=1}^{k} L_{\boldsymbol{\lambda}^{(s)}}\left(b_{s}\right)\right)_{\boldsymbol{\lambda}}^{s i n g}$, see (2.12) , is isomorphic to the algebra $\mathcal{O}_{\boldsymbol{\Lambda}, \boldsymbol{\lambda}, \boldsymbol{b}}$, and that under this isomorphism the $\mathcal{B}_{\boldsymbol{\Lambda}, \boldsymbol{\lambda}, \boldsymbol{b}}$-module $\mathcal{M}_{\boldsymbol{\Lambda}, \boldsymbol{\lambda}, \boldsymbol{b}}$ is isomorphic to the coregular representation of the algebra $\mathcal{O}_{\boldsymbol{\Lambda}, \boldsymbol{\lambda}, \boldsymbol{b}}$.

We begin with a simple observation. Let $A$ be an associative unital algebra, and let $L, M$ be $A$-modules such that $L$ is isomorphic to a subquotient of $M$. Denote 
by $A_{L}$ and $A_{M}$ the images of $A$ in $\operatorname{End}(L)$ and $\operatorname{End}(M)$, respectively, and by $\pi_{L}: A \rightarrow A_{L}, \pi_{M}: A \rightarrow A_{M}$ the corresponding epimorphisms. Then, there exists a unique epimorphism $\pi_{M L}: A_{M} \rightarrow A_{L}$ such that $\pi_{L}=\pi_{M L} \circ \pi_{M}$.

Applying this observation to the Bethe algebra $\mathcal{B}$ and $\mathcal{B}$-modules $\mathcal{M}_{\boldsymbol{\lambda}}, \mathcal{M}_{\boldsymbol{\lambda}, \boldsymbol{a}}$, $\mathcal{M}_{\boldsymbol{\Lambda}, \boldsymbol{\lambda}, \boldsymbol{b}}$, we get a chain of epimorphisms $\mathcal{B} \rightarrow \mathcal{B}_{\boldsymbol{\lambda}} \rightarrow \mathcal{B}_{\boldsymbol{\lambda}, \boldsymbol{a}} \rightarrow \mathcal{B}_{\boldsymbol{\Lambda}, \boldsymbol{\lambda}, \boldsymbol{b}}$. In particular, smaller spaces are naturally modules over Bethe algebras associated to larger spaces.

Let $C_{i}(u), i=1, \ldots, N$, be the Laurent series in $u^{-1}$ obtained by projecting each coefficient of the series $B_{i}(u) \prod_{s=1}^{n}\left(u-z_{s}\right)$ to $\mathcal{B}_{\boldsymbol{\lambda}}$.

Lemma 5.9. The series $C_{i}(u), i=1, \ldots, N, i \leqslant n$, are polynomials in $u$ of degree $n-i$. For $i=n+1, n+2, \ldots, N$, the series $C_{i}(u)$ is zero.

Proof. Lemma 5.9 follows from Theorem 2.1 in [MTV3]. Alternatively, Lemma 5.9 follows from the formula $C_{i}(u)=\tau_{\boldsymbol{\lambda}}\left(G_{i}(u)\right)$.

Let $\bar{C}_{i}(u), i=1, \ldots, N$, be the polynomial obtained by projecting each coefficient of the polynomial $C_{i}(u)$ to $\mathcal{B}_{\boldsymbol{\lambda}, \boldsymbol{a}}$.

Introduce the elements $\bar{C}_{i j s} \in \mathcal{B}_{\boldsymbol{\lambda}, \boldsymbol{a}}$ for $i=1, \ldots, N, j=0,1, \ldots, n-i, s=$ $1, \ldots, k$, by the rule

$$
\sum_{j=0}^{n-i} \bar{C}_{i j s}\left(u-b_{s}\right)^{j}=\bar{C}_{i}(u) .
$$

In addition, let $\bar{C}_{0 j s}, j=0, \ldots, n, s=1, \ldots, k$, be the numbers such that

$$
\sum_{j=0}^{n} \bar{C}_{0 j s}\left(u-b_{s}\right)^{j}=\prod_{r=1}^{k}\left(u-b_{r}\right)^{n_{r}} .
$$

Lemma 5.10. The elements $\bar{C}_{i j s}, i=1, \ldots, N, s=1, \ldots, k, j=0,1, \ldots$, $n_{s}-i-1$, are nilpotent.

Proof. We clearly have $\tau_{\boldsymbol{\lambda}, \boldsymbol{a}} \bar{G}_{i j s}=\bar{C}_{i j s}$. Lemma 5.10 follows from Lemma 4.7 .

Define the indicial polynomial $\bar{\chi}_{s}^{\mathcal{B}}(\alpha)$ at $b_{s}$ by the formula

$$
\bar{\chi}_{s}^{\mathcal{B}}(\alpha)=\sum_{i=0}^{N} \bar{C}_{i, n_{s}-i, s} \prod_{j=0}^{N-i-1}(\alpha-j) .
$$

It is a polynomial of degree $N$ in the variable $\alpha$ with coefficients in $\mathcal{B}_{\boldsymbol{\lambda}, \boldsymbol{a}}$.

Let $\bar{I}_{\boldsymbol{\Lambda}, \boldsymbol{\lambda}, \boldsymbol{b}}^{\mathcal{B}}$ be the ideal in $\mathcal{B}_{\boldsymbol{\lambda}, \boldsymbol{a}}$ generated by the elements $\bar{C}_{i j s}, i=1, \ldots, N$, $s=1, \ldots, k, j=0,1, \ldots, n_{s}-i-1$, and the coefficients of the polynomials

$$
\bar{\chi}_{s}^{\mathcal{B}}(\alpha)-\prod_{\substack{r=1 \\ r \neq s}}^{k}\left(b_{s}-b_{r}\right)^{n_{r}} \prod_{l=1}^{N}\left(\alpha-\lambda_{l}^{(s)}-N+l\right), \quad s=1, \ldots, k .
$$

Lemma 5.11. The ideal $\bar{I}_{\boldsymbol{\Lambda}, \boldsymbol{\lambda}, \boldsymbol{b}}^{\mathcal{B}}$ belongs to the kernel of the projection $\mathcal{B}_{\boldsymbol{\lambda}, \boldsymbol{a}} \rightarrow$ $\mathcal{B}_{\Lambda, \lambda, b}$.

Proof. Set $\bar{C}_{0}(u)=\prod_{s=1}^{k}\left(u-b_{s}\right)^{n_{s}}$. A straightforward calculation shows that the action of the polynomial $\sum_{i=0}^{N} \bar{C}_{i}\left(b_{s}\right) \prod_{j=0}^{N-i-1}(\alpha-j)$ on $\mathcal{M}_{\boldsymbol{\lambda}, \boldsymbol{a}} \subset \bigotimes_{r=1}^{k} L_{\boldsymbol{\lambda}^{(r)}}\left(b_{r}\right)$ coincides with the action of the operator

$$
\prod_{\substack{r=1 \\ r \neq s}}^{k}\left(b_{s}-b_{r}\right)^{n_{r}}\left(1^{\otimes(s-1)} \otimes Z(\alpha-N+1) \otimes 1^{\otimes(k-s)}\right)
$$


cf. MTV2. The lemma follows from Theorem 2.1] and formula (2.3).

Hence, the projection $\mathcal{B}_{\boldsymbol{\lambda}, \boldsymbol{a}} \rightarrow \mathcal{B}_{\boldsymbol{\Lambda}, \boldsymbol{\lambda}, \boldsymbol{b}}$ descends to an epimorphism

$$
\pi_{\boldsymbol{\Lambda}, \boldsymbol{\lambda}, \boldsymbol{b}}: \mathcal{B}_{\boldsymbol{\lambda}, \boldsymbol{a}} / \bar{I}_{\boldsymbol{\Lambda}, \boldsymbol{\lambda}, \boldsymbol{b}}^{\mathcal{B}} \rightarrow \mathcal{B}_{\boldsymbol{\Lambda}, \boldsymbol{\lambda}, \boldsymbol{b}},
$$

which makes $\mathcal{M}_{\boldsymbol{\Lambda}, \boldsymbol{\lambda}, \boldsymbol{b}}$ into a $\mathcal{B}_{\boldsymbol{\lambda}, \boldsymbol{a}} / \bar{I}_{\boldsymbol{\Lambda}, \boldsymbol{\lambda}, \boldsymbol{b}}$-module.

Denote $\operatorname{ker}\left(\bar{I}_{\boldsymbol{\Lambda}, \boldsymbol{\lambda}, \boldsymbol{b}}^{\mathcal{B}}\right)=\left\{v \in \mathcal{M}_{\boldsymbol{\lambda}, \boldsymbol{a}} \mid \bar{I}_{\boldsymbol{\Lambda}, \boldsymbol{\lambda}, \boldsymbol{b}}^{\mathcal{B}} v=0\right\}$. Clearly, $\operatorname{ker}\left(\bar{I}_{\boldsymbol{\Lambda}, \boldsymbol{\lambda}, \boldsymbol{b}}^{\mathcal{B}}\right)$ is a $\mathcal{B}_{\boldsymbol{\lambda}, \boldsymbol{a}}$-submodule of $\mathcal{M}_{\boldsymbol{\lambda}, \boldsymbol{a}}$.

Proposition 5.12. The $\mathcal{B}_{\boldsymbol{\lambda}, \boldsymbol{a}} / \bar{I}_{\boldsymbol{\Lambda}, \boldsymbol{\lambda}, \boldsymbol{b}}^{\mathcal{B}}$-modules $\operatorname{ker}\left(\bar{I}_{\boldsymbol{\Lambda}, \boldsymbol{\lambda}, \boldsymbol{b}}^{\mathcal{B}}\right)$ and $\mathcal{M}_{\boldsymbol{\Lambda}, \boldsymbol{\lambda}, \boldsymbol{b}}$ are isomorphic.

The proposition is proved in Section 5.5 .

Let $\bar{I}_{\boldsymbol{\Lambda}, \boldsymbol{\lambda}, \boldsymbol{b}}^{\mathcal{O}} \subset \mathcal{O}_{\boldsymbol{\lambda}, \boldsymbol{a}}$ be the ideal defined in Section 4.3 Clearly, the map $\tau_{\boldsymbol{\lambda}, \boldsymbol{a}}$ : $\mathcal{O}_{\boldsymbol{\lambda}, \boldsymbol{a}} \rightarrow \mathcal{B}_{\boldsymbol{\lambda}, \boldsymbol{a}}$ sends $\bar{I}_{\boldsymbol{\Lambda}, \boldsymbol{\lambda}, \boldsymbol{b}}^{\mathcal{O}}$ to $\bar{I}_{\boldsymbol{\Lambda}, \boldsymbol{\lambda}, \boldsymbol{b}}^{\mathcal{B}}$. By Lemma 4.8, the maps $\tau_{\boldsymbol{\lambda}, \boldsymbol{a}}$ and $\pi_{\boldsymbol{\Lambda}, \boldsymbol{\lambda}, \boldsymbol{b}}$ induce the homomorphism

$$
\tau_{\Lambda, \boldsymbol{\lambda}, \boldsymbol{b}}: \mathcal{O}_{\boldsymbol{\Lambda}, \boldsymbol{\lambda}, \boldsymbol{b}} \rightarrow \mathcal{B}_{\boldsymbol{\Lambda}, \boldsymbol{\lambda}, \boldsymbol{b}} .
$$

By Theorem 5.8 the map $\mu_{\boldsymbol{\lambda}, \boldsymbol{a}}: \mathcal{O}_{\boldsymbol{\lambda}, \boldsymbol{a}} \rightarrow \mathcal{M}_{\boldsymbol{\lambda}, \boldsymbol{a}}$ sends $\operatorname{Ann}\left(\bar{I}_{\boldsymbol{\Lambda}, \boldsymbol{\lambda}, \boldsymbol{b}}^{\mathcal{O}}\right) \subset \mathcal{O}_{\boldsymbol{\lambda}, \boldsymbol{a}}$ to $\operatorname{ker}\left(\bar{I}_{\boldsymbol{\Lambda}, \boldsymbol{\lambda}, \boldsymbol{b}}^{\mathcal{B}}\right)$. The vector spaces $\operatorname{Ann}\left(\bar{I}_{\boldsymbol{\Lambda}, \boldsymbol{\lambda}, \boldsymbol{b}}^{\mathcal{O}}\right)$ and $\left(\mathcal{O}_{\boldsymbol{\Lambda}, \boldsymbol{\lambda}, \boldsymbol{b}}\right)^{*}$ are isomorphic by Corollary 4.9. Hence, Proposition 5.12 yields that the map $\mu_{\boldsymbol{\lambda}, \boldsymbol{a}}$ induces a bijective linear map

$$
\mu_{\boldsymbol{\Lambda}, \boldsymbol{\lambda}, \boldsymbol{b}}:\left(\mathcal{O}_{\boldsymbol{\Lambda}, \boldsymbol{\lambda}, \boldsymbol{b}}\right)^{*} \rightarrow \mathcal{M}_{\boldsymbol{\Lambda}, \boldsymbol{\lambda}, \boldsymbol{b}} .
$$

For any $F \in \mathcal{O}_{\boldsymbol{\Lambda}, \boldsymbol{\lambda}, \boldsymbol{b}}$, denote by $F^{*} \in \operatorname{End}\left(\left(\mathcal{O}_{\boldsymbol{\Lambda}, \boldsymbol{\lambda}, \boldsymbol{b}}\right)^{*}\right)$ the operator, dual to the operator of multiplication by $F$ on $\mathcal{O}_{\boldsymbol{\Lambda}, \boldsymbol{\lambda}, \boldsymbol{b}}$.

Theorem 5.13. The map $\tau_{\boldsymbol{\Lambda}, \boldsymbol{\lambda}, \boldsymbol{b}}$ is an isomorphism of algebras. For any $F \in \mathcal{O}_{\boldsymbol{\lambda}, \boldsymbol{a}}$ and $G \in\left(\mathcal{O}_{\boldsymbol{\Lambda}, \boldsymbol{\lambda}, \boldsymbol{b}}\right)^{*}$, we have

$$
\mu_{\boldsymbol{\Lambda}, \boldsymbol{\lambda}, \boldsymbol{b}}\left(F^{*} G\right)=\tau_{\boldsymbol{\Lambda}, \boldsymbol{\lambda}, \boldsymbol{b}}(F) \mu_{\boldsymbol{\Lambda}, \boldsymbol{\lambda}, \boldsymbol{b}}(G) .
$$

In other words, the maps $\tau_{\boldsymbol{\Lambda}, \boldsymbol{\lambda}, \boldsymbol{b}}$ and $\mu_{\boldsymbol{\Lambda}, \boldsymbol{\lambda}, \boldsymbol{b}}$ give an isomorphism of the coregular representation of $\mathcal{O}_{\boldsymbol{\Lambda}, \boldsymbol{\lambda}, \boldsymbol{b}}$ on the dual space $\left(\mathcal{O}_{\boldsymbol{\Lambda}, \boldsymbol{\lambda}, \boldsymbol{b}}\right)^{*}$ and the $\mathcal{B}_{\boldsymbol{\Lambda}, \boldsymbol{\lambda}, \boldsymbol{b}}$-module $\mathcal{M}_{\Lambda, \lambda, b}$

Proof. By Lemma 4.8 the isomorphism $\tau_{\boldsymbol{\lambda}, \boldsymbol{a}}: \mathcal{O}_{\boldsymbol{\lambda}, \boldsymbol{a}} \rightarrow \mathcal{B}_{\boldsymbol{\lambda}, \boldsymbol{a}}$ induces the isomorphism

$$
\tau_{\boldsymbol{\Lambda}, \boldsymbol{\lambda}, \boldsymbol{b}}: \mathcal{O}_{\boldsymbol{\Lambda}, \boldsymbol{\lambda}, \boldsymbol{b}} \rightarrow \mathcal{B}_{\boldsymbol{\lambda}, \boldsymbol{a}} / \bar{I}_{\boldsymbol{\Lambda}, \boldsymbol{\lambda}, \boldsymbol{b}}^{\mathcal{B}} .
$$

Therefore the maps $\tau_{\boldsymbol{\Lambda}, \boldsymbol{\lambda}, \boldsymbol{b}}$ and $\mu_{\boldsymbol{\lambda}, \boldsymbol{a}}$ give an isomorphism of the $\mathcal{O}_{\boldsymbol{\Lambda}, \boldsymbol{\lambda}, \boldsymbol{b}}$-module $\operatorname{Ann}\left(\bar{I}_{\boldsymbol{\Lambda}, \boldsymbol{\lambda}, \boldsymbol{b}}^{\mathcal{O}}\right)$ and the $\mathcal{B}_{\boldsymbol{\lambda}, \boldsymbol{a}} / \bar{I}_{\boldsymbol{\Lambda}, \boldsymbol{\lambda}, \boldsymbol{b}}^{\mathcal{B}}$-module $\operatorname{ker}\left(\bar{I}_{\boldsymbol{\Lambda}, \boldsymbol{\lambda}, \boldsymbol{b}}^{\mathcal{B}}\right) ;$ see Theorem 5.8 .

By Lemma 3.8, the $\mathcal{O}_{\boldsymbol{\Lambda}, \boldsymbol{\lambda}, \boldsymbol{b}}$-module $\operatorname{Ann}\left(\bar{I}_{\boldsymbol{\Lambda}, \boldsymbol{\lambda}, \boldsymbol{b}}^{\mathcal{O}}\right)$ is isomorphic to the coregular representation of $\mathcal{O}_{\boldsymbol{\Lambda}, \boldsymbol{\lambda}, \boldsymbol{b}}$ on the dual space $\left(\mathcal{O}_{\boldsymbol{\Lambda}, \boldsymbol{\lambda}, \boldsymbol{b}}\right)^{*}$. In particular, it is faithful. Therefore, the $\mathcal{B}_{\boldsymbol{\lambda}, \boldsymbol{a}} / \bar{I}_{\boldsymbol{\Lambda}, \boldsymbol{\lambda}, \boldsymbol{b}}$-module $\operatorname{ker}\left(\bar{I}_{\boldsymbol{\Lambda}, \boldsymbol{\lambda}, \boldsymbol{b}}^{\mathcal{B}}\right)$ is faithful. By Proposition 5.12 , the $\mathcal{B}_{\boldsymbol{\lambda}, \boldsymbol{a}} / \bar{I}_{\boldsymbol{\Lambda}, \boldsymbol{\lambda}, \boldsymbol{b}}^{\mathcal{B}}$-module $M_{\boldsymbol{\Lambda}, \boldsymbol{\lambda}, \boldsymbol{b}}$, isomorphic to $\operatorname{ker}\left(\bar{I}_{\boldsymbol{\Lambda}, \boldsymbol{\lambda}, \boldsymbol{b}}^{\mathcal{B}}\right)$, is faithful too, which implies that the map $\pi_{\boldsymbol{\Lambda}, \boldsymbol{\lambda}, \boldsymbol{b}}: \mathcal{B}_{\boldsymbol{\lambda}, \boldsymbol{a}} / \bar{I}_{\boldsymbol{\Lambda}, \boldsymbol{\lambda}, \boldsymbol{b}} \rightarrow \mathcal{B}_{\boldsymbol{\Lambda}, \boldsymbol{\lambda}, \boldsymbol{b}}$ is an isomorphism of algebras. The theorem follows.

Remark. By Lemma 4.3, the algebra $\mathcal{O}_{\boldsymbol{\Lambda}, \boldsymbol{\lambda}, \boldsymbol{b}}$ is Frobenius. Therefore, its coregular and regular representations are isomorphic. 
5.5. Proof of Proposition 5.12, We begin the proof with an elementary auxiliary lemma. Let $M$ be a finite-dimensional vector space, $U \subset M$ a subspace, and $E \in \operatorname{End}(M)$.

Lemma 5.14. Let $E M \subset U$ and let the restriction of $E$ to $U$ be invertible in $\operatorname{End}(U)$. Then $E U=U$ and $M=U \oplus \operatorname{ker} E$.

Let $W_{m}$ be the $\mathfrak{g l}_{N}[t]$-module defined in Section 2.3 and let $\boldsymbol{\mu}$ be a partition with at most $N$ parts such that $|\boldsymbol{\mu}|=m$. Recall that $W_{m}$ is a graded vector space, and the grading of $W_{m}$ is defined in Lemma 2.2.

Given a homogeneous vector $w \in\left(W_{m}\right)_{\mu}^{\text {sing }}$, let $\mathcal{L}_{w}(b)$ be the $\mathfrak{g l}_{N}[t]$-submodule of $W_{m}(b)$ generated by the vector $w$. The space $\mathcal{L}_{w}(b)$ is graded. Denote by $\mathcal{L}_{w}^{=}(b)$ and $\mathcal{L}_{w}^{>}(b)$ the subspaces of $\mathcal{L}_{w}(b)$ spanned by homogeneous vectors of degree $\operatorname{deg} w$ and of degree strictly greater than $\operatorname{deg} w$, respectively. The subspace $\mathcal{L}_{w}^{=}(b)$ is a $\mathfrak{g l}_{N}$-submodule of $\mathcal{L}_{w}(b)$ isomorphic to the irreducible $\mathfrak{g l}_{N}$-module $L_{\boldsymbol{\mu}}$. The subspace $\mathcal{L}_{w}^{>}(b)$ is a $\mathfrak{g l}_{N}[t]$-submodule of $\mathcal{L}_{w}(b)$, and the $\mathfrak{g l}_{N}[t]$-module $\mathcal{L}_{w}(b) / \mathcal{L}_{w}^{>}(b)$ is isomorphic to the evaluation module $L_{\boldsymbol{\mu}}(b)$. If $w$ has the largest degree among vectors in $\left(W_{m}\right)_{\mu}^{\text {sing }}$, then $\mathcal{L}_{w}^{>}(b)$, considered as a $\mathfrak{g l}_{N}$-module, does not contain $L_{\boldsymbol{\mu}}$.

For any $s=1, \ldots, k$, pick up a homogeneous vector $w_{s} \in\left(W_{n_{s}}\right)_{\boldsymbol{\lambda}^{(s)}}^{\text {sing }}$ of the largest possible degree. Let $\mathcal{L}_{\boldsymbol{w}}(\boldsymbol{b})$ be the $\mathfrak{g l}_{N}[t]$-submodule of $\bigotimes_{s=1}^{k} W_{n_{s}}\left(b_{s}\right)$ generated by the vector $\bigotimes_{s=1}^{k} w_{s}$.

Denote by $\mathcal{L}_{\boldsymbol{w}}^{=}(\boldsymbol{b})$ and $\mathcal{L}_{\boldsymbol{w}}^{>}(\boldsymbol{b})$ the following subspaces of $\mathcal{L}_{\boldsymbol{w}}(\boldsymbol{b})$ :

$$
\begin{gathered}
\mathcal{L}_{\boldsymbol{w}}^{=}(\boldsymbol{b})=\bigotimes_{s=1}^{k} \mathcal{L}_{w_{s}}^{=}\left(b_{s}\right), \\
\mathcal{L}_{\boldsymbol{w}}^{>}(\boldsymbol{b})=\sum_{s=1}^{k} \mathcal{L}_{w_{1}}\left(b_{1}\right) \otimes \cdots \otimes \mathcal{L}_{w_{s}}^{>}\left(b_{s}\right) \otimes \cdots \otimes \mathcal{L}_{w_{k}}\left(b_{k}\right) .
\end{gathered}
$$

The subspace $\mathcal{L}_{\boldsymbol{w}}^{>}(\boldsymbol{b})$ is a $\mathfrak{g l}_{N}[t]$-submodule of $\mathcal{L}_{\boldsymbol{w}}(\boldsymbol{b})$, and the $\mathfrak{g l}_{N}[t]$-module $\mathcal{L}_{\boldsymbol{w}}(\boldsymbol{b}) / \mathcal{L}_{\boldsymbol{w}}^{>}(\boldsymbol{b})$ is isomorphic to the tensor product of evaluation modules $\bigotimes_{s=1}^{k} L_{\boldsymbol{\lambda}^{(s)}}\left(b_{s}\right)$.

Recall that the space $\bigotimes_{s=1}^{k} W_{n_{s}}$ has the second $\mathfrak{g l}_{N}[t]$-module structure, denoted $\operatorname{gr}\left(\otimes_{s=1}^{k} W_{n_{s}}\left(b_{s}\right)\right)$, which was introduced at the end of Section 2.3. The subspace $\mathcal{L}_{\boldsymbol{w}}^{>}(\boldsymbol{b})$ is a $\mathfrak{g l}_{N}[t]$-submodule of $\operatorname{gr}\left(\bigotimes_{s=1}^{k} W_{n_{s}}\left(b_{s}\right)\right)$, isomorphic to a direct sum of irreducible $\mathfrak{g l}_{N}[t]$-modules of the form $\bigotimes_{s=1}^{k} L_{\boldsymbol{\mu}^{(s)}}\left(b_{s}\right)$, where $\left|\boldsymbol{\mu}^{(s)}\right|=n_{s}, \quad s=$ $1, \ldots, k$, see Lemmas 2.5 and 2.6, and $\left(\boldsymbol{\mu}^{(1)}, \ldots, \boldsymbol{\mu}^{(k)}\right) \neq\left(\boldsymbol{\lambda}^{(1)}, \ldots, \boldsymbol{\lambda}^{(k)}\right)$ for any term of the sum.

The subspace $\mathcal{M}_{\boldsymbol{\lambda}, \boldsymbol{a}}=\left(\otimes_{s=1}^{k} W_{n_{s}}\left(b_{s}\right)\right)_{\lambda}^{\text {sing }}$ is invariant under the action of the Bethe algebra $\mathcal{B} \subset U\left(\mathfrak{g l}_{N}[t]\right)$. This makes it into a $\mathcal{B}$-module, which we call the standard $\mathcal{B}$-module structure on $\mathcal{M}_{\boldsymbol{\lambda}, \boldsymbol{a}}$. The $\mathcal{B}$-module $\mathcal{M}_{\boldsymbol{\lambda}, \boldsymbol{a}}$ contains the submodules $\mathcal{M}_{\boldsymbol{\Lambda}, \boldsymbol{\lambda}, \boldsymbol{b}}^{\boldsymbol{w}}=\left(\mathcal{L}_{\boldsymbol{w}}(\boldsymbol{b})\right)_{\boldsymbol{\lambda}}^{\text {sing }}$ and $\mathcal{M}_{\boldsymbol{\Lambda}, \boldsymbol{\lambda}, \boldsymbol{b}}^{\boldsymbol{w},>}=\left(\mathcal{L}_{\boldsymbol{w}}^{>}(\boldsymbol{b})\right)_{\boldsymbol{\lambda}}^{\text {sing }}$, and the subspace $\mathcal{M}_{\boldsymbol{\Lambda}, \boldsymbol{\lambda}, \boldsymbol{b}}^{\boldsymbol{w},=}=\left(\mathcal{L}_{\boldsymbol{w}}^{=}(\boldsymbol{b})\right)_{\boldsymbol{\lambda}}^{\text {sing }}$. As vector spaces, $\mathcal{M}_{\boldsymbol{\Lambda}, \boldsymbol{\lambda}, \boldsymbol{b}}^{\boldsymbol{w}}=\mathcal{M}_{\boldsymbol{\Lambda}, \boldsymbol{\lambda}, \boldsymbol{b}}^{\boldsymbol{w},=} \oplus \mathcal{M}_{\boldsymbol{\Lambda}, \boldsymbol{\lambda}, \boldsymbol{b}}^{\boldsymbol{w},>}$. The $\mathcal{B}$-modules $\mathcal{M}_{\boldsymbol{\Lambda}, \boldsymbol{\lambda}, \boldsymbol{b}}^{\boldsymbol{w}} / \mathcal{M}_{\boldsymbol{\Lambda}, \boldsymbol{\lambda}, \boldsymbol{b}}^{\boldsymbol{w}, \boldsymbol{s}}$ and $\mathcal{M}_{\boldsymbol{\Lambda}, \boldsymbol{\lambda}, \boldsymbol{b}}$ are isomorphic.

The space $\mathcal{M}_{\boldsymbol{\lambda}, \boldsymbol{a}}$ has another $\mathcal{B}$-module structure, inherited from the $\mathfrak{g l}_{N}[t]$ module structure of $\operatorname{gr}\left(\bigotimes_{s=1}^{k} W_{n_{s}}\left(b_{s}\right)\right)$. We denote the new structure gr $\mathcal{M}_{\boldsymbol{\lambda}, \boldsymbol{a}}$. The subspaces $\mathcal{M}_{\boldsymbol{\Lambda}, \boldsymbol{\lambda}, \boldsymbol{b}}^{\boldsymbol{w}}, \mathcal{M}_{\boldsymbol{\Lambda}, \boldsymbol{\lambda}, \boldsymbol{b}}^{\boldsymbol{w},=}, \mathcal{M}_{\boldsymbol{\Lambda}, \boldsymbol{\lambda}, \boldsymbol{b}}^{\boldsymbol{w},>}$ are $\mathcal{B}$-submodules of the $\mathcal{B}$-module $\operatorname{gr} \mathcal{M}_{\boldsymbol{\lambda}, \boldsymbol{a}}$. The submodule $\mathcal{M}_{\boldsymbol{\Lambda}, \boldsymbol{\lambda}, \boldsymbol{b}}^{\boldsymbol{w},=} \subset \operatorname{gr} \mathcal{M}_{\boldsymbol{\lambda}, \boldsymbol{a}}$ is isomorphic to the $\mathcal{B}$-module 
$\mathcal{M}_{\boldsymbol{\Lambda}, \boldsymbol{\lambda}, \boldsymbol{b}}$, and the submodule $\mathcal{M}_{\boldsymbol{\Lambda}, \boldsymbol{\lambda}, \boldsymbol{b}}^{\boldsymbol{w},>} \subset \operatorname{gr} \mathcal{M}_{\boldsymbol{\lambda}, \boldsymbol{a}}$ is isomorphic to a direct sum of $\mathcal{B}$-modules of the form $\mathcal{M}_{\mathbf{M}, \boldsymbol{\lambda}, \boldsymbol{b}}$, where $\mathbf{M}=\left(\boldsymbol{\mu}^{(1)}, \ldots, \boldsymbol{\mu}^{(k)}\right),\left|\boldsymbol{\mu}^{(s)}\right|=n_{s}$, $s=1, \ldots, k$, and $\mathbf{M} \neq \boldsymbol{\Lambda}$ for any term of the sum.

In the picture described above, we can regard all $\mathcal{B}$-modules involved as $\mathcal{B}_{\boldsymbol{\lambda}, a^{-}}$ modules.

For any $F \in \mathcal{B}_{\boldsymbol{\lambda}, \boldsymbol{a}}$, we denote by $\operatorname{gr} F \in \operatorname{End}\left(\mathcal{M}_{\boldsymbol{\lambda}, \boldsymbol{a}}\right)$ the linear operator corresponding to the action of $F$ on $\operatorname{gr} \mathcal{M}_{\boldsymbol{\lambda}, \boldsymbol{a}}$. The map $F \mapsto \operatorname{gr} F$ is an algebra homomorphism.

Let complex numbers $c_{1}, \ldots, c_{k}, \alpha_{1}, \ldots, \alpha_{k}$ be such that

$$
\sum_{s=1}^{k} c_{s}\left(\prod_{i=1}^{N}\left(\alpha_{s}-\mu_{i}^{(s)}-N+i\right)-\prod_{i=1}^{N}\left(\alpha_{s}-\lambda_{i}^{(s)}-N+i\right)\right) \neq 0,
$$

for any sequence of partitions $\left(\boldsymbol{\mu}^{(1)}, \ldots, \boldsymbol{\mu}^{(k)}\right) \neq\left(\boldsymbol{\lambda}^{(1)}, \ldots, \boldsymbol{\lambda}^{(k)}\right)$. Let

$$
E=\sum_{s=1}^{k} c_{s}\left(\bar{\chi}_{s}^{\mathcal{B}}\left(\alpha_{s}\right)-\prod_{\substack{r=1 \\ r \neq s}}^{k}\left(b_{s}-b_{r}\right)^{n_{r}} \prod_{i=1}^{N}\left(\alpha_{s}-\lambda_{i}^{(s)}-N+i\right)\right) \in \bar{I}_{\boldsymbol{\Lambda}, \boldsymbol{\lambda}, \boldsymbol{b}}^{\mathcal{B}},
$$

where $\bar{\chi}_{s}^{\mathcal{B}}$ is the indicial polynomial (5.6). With respect to the standard $\mathcal{B}$-module structure on $\mathcal{M}_{\boldsymbol{\lambda}, \boldsymbol{a}}$, we have $E \mathcal{M}_{\boldsymbol{\Lambda}, \boldsymbol{\lambda}, \boldsymbol{b}}^{\boldsymbol{w}} \subset \mathcal{M}_{\boldsymbol{\Lambda}, \boldsymbol{\lambda}, \boldsymbol{b}}^{\boldsymbol{w},}$.

Lemma 5.15. The restriction of $E$ to $\mathcal{M}_{\boldsymbol{\Lambda}, \boldsymbol{\lambda}, \boldsymbol{b}}^{\boldsymbol{w},>}$ is invertible in $\operatorname{End}\left(\mathcal{M}_{\boldsymbol{\Lambda}, \boldsymbol{\lambda}, \boldsymbol{b}}^{\boldsymbol{w},>}\right)$.

Proof. Formula (5.7) implies that the projection of $E$ to $\mathcal{B}_{\boldsymbol{\Lambda}, \boldsymbol{\lambda}, \boldsymbol{b}}$ equals zero, and the projection of $E$ to $\mathcal{B}_{\mathbf{M}, \boldsymbol{\lambda}, \boldsymbol{b}}$ with $\mathbf{M} \neq \boldsymbol{\Lambda}$ is invertible. This means that the restriction of the operator gr $E$ to $\mathcal{M}_{\boldsymbol{\Lambda}, \boldsymbol{\lambda}, \boldsymbol{b}}^{\boldsymbol{w},>}$ is invertible in $\operatorname{End}\left(\mathcal{M}_{\boldsymbol{\Lambda}, \boldsymbol{\lambda}, \boldsymbol{b}}^{\boldsymbol{w},>}\right)$. Therefore, the restriction of $E$ to $\mathcal{M}_{\boldsymbol{\Lambda}, \boldsymbol{\lambda}, \boldsymbol{b}}^{\boldsymbol{w},>}$ is invertible in $\operatorname{End}\left(\mathcal{M}_{\boldsymbol{\Lambda}, \boldsymbol{\lambda}, \boldsymbol{b}}^{\boldsymbol{w},>}\right)$.

Denote $\operatorname{ker}_{\boldsymbol{\Lambda}, \boldsymbol{\lambda}, \boldsymbol{b}}^{\boldsymbol{w}} E=\operatorname{ker} E \cap \mathcal{M}_{\boldsymbol{\Lambda}, \boldsymbol{\lambda}, \boldsymbol{b}}^{\boldsymbol{w}}$. By Lemma 5.14 , the canonical projection

$$
\mathcal{M}_{\Lambda, \lambda, b}^{w} \rightarrow \mathcal{M}_{\Lambda, \lambda, b}^{w} / \mathcal{M}_{\Lambda, \lambda, b}^{w,>} \simeq \mathcal{M}_{\Lambda, \lambda, b}
$$

induces an isomorphism $\operatorname{ker}_{\boldsymbol{\Lambda}, \boldsymbol{\lambda}, \boldsymbol{b}}^{\boldsymbol{w}} E \rightarrow \mathcal{M}_{\boldsymbol{\Lambda}, \boldsymbol{\lambda}, \boldsymbol{b}}$ of vector spaces. Since the algebra $\mathcal{B}_{\boldsymbol{\lambda}, \boldsymbol{a}}$ is commutative, the subspace $\operatorname{ker}_{\boldsymbol{\Lambda}, \boldsymbol{\lambda}, \boldsymbol{b}}^{\boldsymbol{w}} E$ is a $\mathcal{B}$-submodule, and the map $\operatorname{ker}_{\boldsymbol{\Lambda}, \boldsymbol{\lambda}, \boldsymbol{b}}^{\boldsymbol{w}} E \rightarrow \mathcal{M}_{\boldsymbol{\Lambda}, \boldsymbol{\lambda}, \boldsymbol{b}}$ is an isomorphism of $\mathcal{B}_{\boldsymbol{\lambda}, \boldsymbol{a}}$-modules.

Lemma 5.11 implies that elements of the ideal $\bar{I}_{\boldsymbol{\Lambda}, \boldsymbol{\lambda}, \boldsymbol{b}}^{\mathcal{B}}$ act on $\mathcal{M}_{\boldsymbol{\Lambda}, \boldsymbol{\lambda}, \boldsymbol{b}}$ by zero. Hence, they act by zero on $\operatorname{ker}_{\boldsymbol{\Lambda}, \boldsymbol{\lambda}, \boldsymbol{b}}^{\boldsymbol{w}} E$, that is, $\operatorname{ker}_{\boldsymbol{\Lambda}, \boldsymbol{\lambda}, \boldsymbol{b}}^{\boldsymbol{w}} E \subset \operatorname{ker}\left(\bar{I}_{\boldsymbol{\Lambda}, \boldsymbol{\lambda}, \boldsymbol{b}}^{\mathcal{B}}\right)$. On the other hand, we have

$$
\begin{aligned}
\operatorname{dim} \operatorname{ker}\left(\bar{I}_{\boldsymbol{\Lambda}, \boldsymbol{\lambda}, \boldsymbol{b}}^{\mathcal{B}}\right) & =\operatorname{dim} \operatorname{Ann}\left(\bar{I}_{\boldsymbol{\Lambda}, \boldsymbol{\lambda}, \boldsymbol{b}}^{\mathcal{O}}\right) \\
& =\operatorname{dim} \mathcal{O}_{\boldsymbol{\Lambda}, \boldsymbol{\lambda}, \boldsymbol{b}}=\operatorname{dim} \mathcal{M}_{\boldsymbol{\Lambda}, \boldsymbol{\lambda}, \boldsymbol{b}}=\operatorname{dim} \operatorname{ker}_{\boldsymbol{\Lambda}, \boldsymbol{\lambda}, \boldsymbol{b}}^{\boldsymbol{w}} E
\end{aligned}
$$

see Theorem [5.8, Corollary 4.9 and formula (4.4), which yields $\operatorname{ker}_{\boldsymbol{\Lambda}, \boldsymbol{\lambda}, \boldsymbol{b}}^{\boldsymbol{w}} E=$ $\operatorname{ker}\left(\bar{I}_{\boldsymbol{\Lambda}, \boldsymbol{\lambda}, \boldsymbol{b}}^{\mathcal{B}}\right)$. Proposition 5.12 is proved.

Remark. Note that formula (4.4) is an important ingredient of the proof. 


\section{Applichtions}

In this section we state some of the obtained results in a way that is relatively independent from the main part of the paper. For convenience, we recall some definitions and facts.

The Bethe algebra $\mathcal{B}$ is a commutative subalgebra of $U\left(\mathfrak{g l}_{N}[t]\right)$, defined in Section 2.7. It is generated by the elements $B_{i j}, i=1, \ldots, N, j \in \mathbb{Z}_{\geqslant i}$, which are coefficients of the series $B_{i}(u), i=1, \ldots, N$; see formulae (2.8), (2.9).

If $M$ is a $\mathcal{B}$-module and $\xi: \mathcal{B} \rightarrow \mathbb{C}$ a homomorphism, then the eigenspace of the $\mathcal{B}$-action on $M$ corresponding to $\xi$ is defined as $\bigcap_{B \in \mathcal{B}} \operatorname{ker}\left(\left.B\right|_{M}-\xi(B)\right)$ and the generalized eigenspace of the $\mathcal{B}$-action on $M$ corresponding to $\xi$ is defined as $\bigcap_{B \in \mathcal{B}}\left(\bigcup_{m=1}^{\infty} \operatorname{ker}\left(\left.B\right|_{M}-\xi(B)\right)^{m}\right)$.

6.1. Tensor products of irreducible modules. For a partition $\boldsymbol{\lambda}$ with at most $N$ parts, $L_{\boldsymbol{\lambda}}$ is the irreducible finite-dimensional $\mathfrak{g l}_{N}$-module of highest weight $\boldsymbol{\lambda}$.

Let $\boldsymbol{\lambda}^{(1)}, \ldots, \boldsymbol{\lambda}^{(k)}$ be partitions with at most $N$ parts, $b_{1}, \ldots, b_{k}$ distinct complex numbers. We are interested in the action of the Bethe algebra $\mathcal{B}$ on the tensor product $\bigotimes_{s=1}^{k} L_{\boldsymbol{\lambda}^{(s)}}\left(b_{s}\right)$ of evaluation $\mathfrak{g l}_{N}[t]$-modules.

Since $\mathcal{B}$ commutes with the subalgebra $U\left(\mathfrak{g l}_{N}\right) \subset U\left(\mathfrak{g l}_{N}[t]\right)$, the action of $\mathcal{B}$ preserves the subspace of singular vectors $\left(\bigotimes_{s=1}^{k} L_{\boldsymbol{\lambda}^{(s)}}\left(b_{s}\right)\right)^{\text {sing }}$ as well as the weight subspaces of $\bigotimes_{s=1}^{k} L_{\boldsymbol{\lambda}^{(s)}}\left(b_{s}\right)$. The action of $\mathcal{B}$ on $\bigotimes_{s=1}^{k} L_{\boldsymbol{\lambda}^{(s)}}\left(b_{s}\right)$ is determined by the action of $\mathcal{B}$ on $\left(\bigotimes_{s=1}^{k} L_{\boldsymbol{\lambda}^{(s)}}\left(b_{s}\right)\right)^{\text {sing }}$.

Denote $\boldsymbol{\Lambda}=\left(\boldsymbol{\lambda}^{(1)}, \ldots, \boldsymbol{\lambda}^{(k)}\right)$. Given a partition $\boldsymbol{\lambda}$ with at most $N$ parts such that $|\boldsymbol{\lambda}|=\sum_{s=1}^{k}\left|\boldsymbol{\lambda}^{(s)}\right|$, let $\Delta_{\boldsymbol{\Lambda}, \boldsymbol{\lambda}, \boldsymbol{b}}$ be the set of all monic Fuchsian differential operators of order $N$,

$$
\mathcal{D}=\partial^{N}+\sum_{i=1}^{N} h_{i}^{\mathcal{D}}(u) \partial^{N-i},
$$

where $\partial=d / d u$, with the following properties.

(a) The singular points of $\mathcal{D}$ are at $b_{1}, \ldots, b_{k}$ and $\infty$ only.

(b) The exponents of $\mathcal{D}$ at $b_{s}, s=1, \ldots, k$, are equal to $\lambda_{N}^{(s)}, \lambda_{N-1}^{(s)}+1$, $\ldots, \lambda_{1}^{(s)}+N-1$.

(c) The exponents of $\mathcal{D}$ at $\infty$ are equal to $1-N-\lambda_{1}, 2-N-\lambda_{2}, \ldots,-\lambda_{N}$.

(d) The operator $\mathcal{D}$ is monodromy free.

Equivalently, the last property can be replaced by

(e) The kernel of the operator $\mathcal{D}$ consists of polynomials only.

A differential operator $\mathcal{D}$ belongs to the set $\Delta_{\boldsymbol{\Lambda}, \boldsymbol{\lambda}, \boldsymbol{b}}$ if and only if the kernel of $\mathcal{D}$ is a point of the intersection of Schubert cells $\Omega_{\boldsymbol{\Lambda}, \overline{\boldsymbol{\lambda}}, \boldsymbol{b}}$; see Lemma 4.1.

Denote $n_{s}=\left|\boldsymbol{\lambda}^{(s)}\right|, s=1, \ldots, k$, and $n=\sum_{s=1}^{k} n_{s}$.

Theorem 6.1. The action of the Bethe algebra $\mathcal{B}$ on $\left(\bigotimes_{s=1}^{k} L_{\boldsymbol{\lambda}^{(s)}}\left(b_{s}\right)\right)^{\text {sing }}$ has the following properties.

(i) For every $i=1, \ldots, N, i \leqslant n$, the action of the series $B_{i}(u)$ is given by the power series expansion in $u^{-1}$ of a rational function of the form $A_{i}(u) \prod_{s=1}^{k}\left(u-b_{s}\right)^{-n_{s}}$, where $A_{i}(u)$ is a polynomial of degree $n-i$ with coefficients in $\operatorname{End}\left(\left(\bigotimes_{s=1}^{k} L_{\boldsymbol{\lambda}^{(s)}}\right)^{\text {sing }}\right)$. For $i=n+1, n+2, \ldots, N$, the series $B_{i}(u)$ acts by zero. 
(ii) The image of $\mathcal{B}$ in $\operatorname{End}\left(\left(\bigotimes_{s=1}^{k} L_{\boldsymbol{\lambda}^{(s)}}\right)^{\text {sing }}\right)$ is a maximal commutative subalgebra of dimension $\operatorname{dim}\left(\bigotimes_{s=1}^{k} L_{\boldsymbol{\lambda}^{(s)}}\right)^{\text {sing }}$.

(iii) Each eigenspace of the action of $\mathcal{B}$ is one-dimensional.

(iv) Each generalized eigenspace of the action of $\mathcal{B}$ is generated over $\mathcal{B}$ by one vector.

(v) The eigenspaces of the action of $\mathcal{B}$ on $\left(\bigotimes_{s=1}^{k} L_{\boldsymbol{\lambda}^{(s)}}\left(b_{s}\right)\right)_{\boldsymbol{\lambda}}^{\text {sing }}$ are in a oneto-one correspondence with differential operators from $\Delta_{\boldsymbol{\Lambda}, \boldsymbol{\lambda}, \boldsymbol{b}}$. Moreover, if $\mathcal{D}$ is the differential operator, corresponding to an eigenspace, then the coefficients of the series $h_{i}^{\mathcal{D}}(u)$ are the eigenvalues of the action of the respective coefficients of the series $B_{i}(u)$.

(vi) The eigenspaces of the action of $\mathcal{B}$ on $\left(\bigotimes_{s=1}^{k} L_{\boldsymbol{\lambda}^{(s)}}\left(b_{s}\right)\right)_{\boldsymbol{\lambda}}^{\text {sing }}$ are in a one-toone correspondence with points of the intersection of Schubert cells $\Omega_{\boldsymbol{\Lambda}, \overline{\boldsymbol{\lambda}}, \boldsymbol{b}}$, given by (4.1).

Proof. The first property follows from Lemma 5.9. The other properties follow from Theorem 5.13, Lemma 4.1. and standard facts about the coregular representations of Frobenius algebras given in Section 3.3

The intersection of Schubert cells $\Omega_{\boldsymbol{\Lambda}, \overline{\boldsymbol{\lambda}}, \boldsymbol{b}}$ is transversal if the scheme-theoretic intersection $\mathcal{O}_{\boldsymbol{\Lambda}, \boldsymbol{\lambda}, \boldsymbol{b}}$ is a direct sum of one-dimensional algebras.

Corollary 6.2. The following statements are equivalent.

(i) The action of the Bethe algebra $\mathcal{B}$ on $\left(\bigotimes_{s=1}^{k} L_{\boldsymbol{\lambda}^{(s)}}\left(b_{s}\right)\right)_{\boldsymbol{\lambda}}^{\text {sing }}$ is diagonalizable.

(ii) The set $\Delta_{\boldsymbol{\Lambda}, \boldsymbol{\lambda}, \boldsymbol{b}}$ consists of $\operatorname{dim}\left(\bigotimes_{s=1}^{k} L_{\boldsymbol{\lambda}(s)}\right)_{\boldsymbol{\lambda}}^{\text {sing }}$ distinct points.

(iii) The set $\Omega_{\boldsymbol{\Lambda}, \overline{\boldsymbol{\lambda}}, \boldsymbol{b}}$ consists of $\operatorname{dim}\left(\bigotimes_{s=1}^{k} L_{\boldsymbol{\lambda}(s)}\right)_{\boldsymbol{\lambda}}^{\text {sing }}$ distinct points.

(iv) The intersection of Schubert cells $\Omega_{\boldsymbol{\Lambda}, \overline{\boldsymbol{\lambda}}, \boldsymbol{b}}$ is transversal.

We call a differential operator $\mathcal{D} \in \Delta_{\boldsymbol{\Lambda}, \boldsymbol{\lambda}, \boldsymbol{b}}$ real if all $h_{i}^{\mathcal{D}}(u)$ are rational functions with real coefficients. We call $X \in \Omega_{\boldsymbol{\Lambda}, \overline{\boldsymbol{\lambda}}, \boldsymbol{b}}$ real if $X$ has a basis consisting of polynomials with real coefficients.

Corollary 6.3. Let $b_{1}, \ldots, b_{k}$ be distinct real numbers.

(i) The set $\Delta_{\boldsymbol{\Lambda}, \boldsymbol{\lambda}, \boldsymbol{b}}$ consists of $\operatorname{dim}\left(\bigotimes_{s=1}^{k} L_{\boldsymbol{\lambda}}(s)\right)_{\boldsymbol{\lambda}}^{\text {sing }}$ distinct real points.

(ii) The intersection of Schubert cells $\Omega_{\boldsymbol{\Lambda}, \overline{\boldsymbol{\lambda}}, \boldsymbol{b}}$ consists of $\operatorname{dim}\left(\bigotimes_{s=1}^{k} L_{\boldsymbol{\lambda}^{(s)}}\right)_{\boldsymbol{\lambda}}^{\text {sing }}$ distinct real points and is transversal.

Proof. Denote by $\mathcal{B}^{\mathbb{R}}$ and $L_{\lambda}^{\mathbb{R}}$ the real forms of the Bethe algebra $\mathcal{B}$ and the module $L_{\boldsymbol{\lambda}}$, respectively. The real vector space $\left(\bigotimes_{s=1}^{k} L_{\boldsymbol{\lambda}^{(s)}}^{\mathbb{R}}\right)_{\boldsymbol{\lambda}}^{\text {sing }}$ has a natural positive definite bilinear form, which comes from the tensor product of the Shapovalov forms on the tensor factors. If $b_{1}, \ldots, b_{k}$ are distinct real numbers, and $B \in \mathcal{B}^{\mathbb{R}}$, then $B$ acts on $\left(\bigotimes_{s=1}^{k} L_{\boldsymbol{\lambda}^{(s)}}^{\mathbb{R}}\left(b_{s}\right)\right)_{\boldsymbol{\lambda}}^{\text {sing }}$ as a linear operator, symmetric with respect to that form; see MTV1, MTV2. In particular $B$ is diagonalizable and all its eigenvalues are real. The corollary follows.

For $N=2$, this corollary is obtained in [EG].

6.2. Weyl modules. Let $n_{1}, \ldots, n_{k}$ be natural numbers and $b_{1}, \ldots, b_{k}$ distinct complex numbers. Let $\bigotimes_{s=1}^{k} W_{n_{s}}\left(b_{s}\right)$ be the Weyl module associated to $\boldsymbol{n}, \boldsymbol{b}$ defined in Section 2.3. We are interested in the action of the Bethe algebra $\mathcal{B}$ on the Weyl module $\bigotimes_{s=1}^{k} W_{n_{s}}\left(b_{s}\right)$. 
The action of $\mathcal{B}$ preserves the subspace of singular vectors $\left(\bigotimes_{s=1}^{k} W_{n_{s}}\left(b_{s}\right)\right)^{\text {sing }}$ as well as the weight subspaces of $\bigotimes_{s=1}^{k} W_{n_{s}}\left(b_{s}\right)$. The action of $\mathcal{B}$ on $\bigotimes_{s=1}^{k} W_{n_{s}}\left(b_{s}\right)$ is determined by the action of $\mathcal{B}$ on $\left(\bigotimes_{s=1}^{k} W_{n_{s}}\left(b_{s}\right)\right)^{\text {sing }}$.

Recall that $V$ denotes the irreducible $\mathfrak{g l}_{N}$-module of highest weight $(1,0, \ldots, 0)$, which is the vector representation of $\mathfrak{g l}_{N}$. Set $n=n_{1}+\cdots+n_{k}$.

Denote by $\Delta_{\boldsymbol{n}, \boldsymbol{b}}$ the set of all monic differential operators $\mathcal{D}$ of order $N$ with the following properties.

(a) The kernel of the operator $\mathcal{D}$ consists of polynomials only.

(b) The first coefficient $h_{1}^{\mathcal{D}}(u)$ of $\mathcal{D}$, see (6.1), is equal to $-\sum_{s=1}^{k} n_{s}\left(u-b_{s}\right)^{-1}$. If $\mathcal{D} \in \Delta_{\boldsymbol{n}, \boldsymbol{b}}$, then $\mathcal{D}$ is a Fuchsian differential operator with singular points at $b_{1}, \ldots, b_{k}$ and $\infty$ only.

Denote by $\mathrm{Wr}_{\boldsymbol{n}, \boldsymbol{b}}^{-1}$ the set of all $N$-dimensional subspaces of $\mathbb{C}[u]$, admitting a basis $p_{1}(u), \ldots, p_{N}(u)$ with the Wronskian $\operatorname{Wr}\left(p_{1}(u), \ldots, p_{N}(u)\right)=\prod_{s=1}^{k}\left(u-b_{s}\right)^{n_{s}}$. A differential operator $\mathcal{D}$ belongs to the set $\Delta_{\boldsymbol{n}, \boldsymbol{b}}$ if and only if the kernel of $\mathcal{D}$ belongs to the set $\mathrm{Wr}_{\boldsymbol{n}, \boldsymbol{b}}^{-1}$.

Theorem 6.4. The action of the Bethe algebra $\mathcal{B}$ on $\left(\bigotimes_{s=1}^{k} W_{n_{s}}\left(b_{s}\right)\right)^{\text {sing }}$ has the following properties.

(i) For every $i=1, \ldots, N, i \leqslant n$, the action of the series $B_{i}(u)$ is given by the power series expansion in $u^{-1}$ of a rational function of the form $A_{i}(u) \prod_{s=1}^{k}\left(u-b_{s}\right)^{-n_{s}}$, where $A_{i}(u)$ is a polynomial of degree $n-i$ with coefficients in $\operatorname{End}\left(\left(\bigotimes_{s=1}^{k} W_{n_{s}}\right)^{\text {sing }}\right)$. For $i=n+1, n+2, \ldots, N$, the series $B_{i}(u)$ acts by zero.

(ii) The image of $\mathcal{B}$ in $\operatorname{End}\left(\left(\bigotimes_{s=1}^{k} W_{n_{s}}\right)^{\text {sing }}\right)$ is a maximal commutative subalgebra of dimension $\operatorname{dim}\left(V^{\otimes n}\right)^{\text {sing }}$.

(iii) Each eigenspace of the action of $\mathcal{B}$ is one-dimensional.

(iv) Each generalized eigenspace of the action of $\mathcal{B}$ is generated over $\mathcal{B}$ by one vector.

(v) The eigenspaces of the action of $\mathcal{B}$ on $\left(\bigotimes_{s=1}^{k} W_{n_{s}}\left(b_{s}\right)\right)^{\text {sing }}$ are in a oneto-one correspondence with differential operators from $\Delta_{\boldsymbol{n}, \boldsymbol{b}}$. Moreover, if $\mathcal{D}$ is the differential operator, corresponding to an eigenspace, then the coefficients of the series $h_{i}^{\mathcal{D}}(u)$ are the eigenvalues of the action of the respective coefficients of the series $B_{i}(u)$.

(vi) The eigenspaces of the action of $\mathcal{B}$ on $\left(\bigotimes_{s=1}^{k} W_{n_{s}}\left(b_{s}\right)\right)^{\text {sing }}$ are in a one-toone correspondence with spaces of polynomials from $\mathrm{Wr}_{\boldsymbol{n}, \boldsymbol{b}}^{-1}$.

Proof. The first property follows from Lemmas 2.13 and 5.9. The other properties follow from Theorem 5.8 formulae (3.5) and (5.2), and standard facts about the regular representations of Frobenius algebras given in Section 3.3

Corollary 6.5. The following three statements are equivalent.

(i) The action of the Bethe algebra $\mathcal{B}$ on $\left(\bigotimes_{s=1}^{k} W_{n_{s}}\left(b_{s}\right)\right)^{\text {sing }}$ is diagonalizable.

(ii) The set $\Delta_{\boldsymbol{n}, \boldsymbol{b}}$ consists of $\operatorname{dim}\left(V^{\otimes n}\right)^{\text {sing }}$ distinct points.

(iii) The set $\mathrm{Wr}_{\boldsymbol{n}, \boldsymbol{b}}^{-1}$ consists of $\operatorname{dim}\left(V^{\otimes n}\right)^{\text {sing }}$ distinct points.

Remark. It is easy to see that the set $\Delta_{\boldsymbol{n}, \boldsymbol{b}}$ is a disjoint union of the sets $\Delta_{\boldsymbol{\Lambda}, \boldsymbol{\lambda}, \boldsymbol{b}}$ with $\left|\boldsymbol{\lambda}^{(s)}\right|=n_{s}, s=1, \ldots, k$. Similarly, the set $\mathrm{Wr}_{\boldsymbol{n}, \boldsymbol{b}}^{-1}$ is a disjoint union of the 
sets $\Omega_{\boldsymbol{\Lambda}, \overline{\boldsymbol{\lambda}}, \boldsymbol{b}}$ with $\left|\boldsymbol{\lambda}^{(s)}\right|=n_{s}, \quad s=1, \ldots, k$. Comparing items (ii), (iii) of Corollaries 6.5 and 6.2, one can see that the sets $\Delta_{n, \boldsymbol{b}}$ and $\mathrm{Wr}_{\boldsymbol{n}, \boldsymbol{b}}^{-1}$ can have cardinality $\operatorname{dim}\left(V^{\otimes n}\right)^{\operatorname{sing}}$ only if for each $s=1, \ldots, k$ the decomposition of the $\mathfrak{g l}_{N}$-module $V^{\otimes n_{s}}$ into the direct sum of irreducible modules is multiplicity free, that is, each of the numbers $n_{1}, \ldots, n_{k}$ equals 1 or 2 .

6.3. Monodromy free Fuchsian differential operators. In this section we give a reformulation of a part of Corollary 6.3

Let $\boldsymbol{\mu}^{(s)}=\left(\mu_{1}^{(s)}, \ldots, \mu_{N}^{(s)}\right), s=0, \ldots, k$, be sequences of nonincreasing integers, $\mu_{1}^{(s)} \geqslant \cdots \geqslant \mu_{N}^{(s)}$, and let $\boldsymbol{b}=\left(b_{0}, \ldots, b_{k}\right)$ be a sequence of distinct points on the Riemann sphere. Set $\mathbf{M}=\left(\boldsymbol{\mu}^{(0)}, \ldots, \boldsymbol{\mu}^{(k)}\right)$.

Denote by $\Delta_{\mathbf{M}, \boldsymbol{b}}$ the set of all monic Fuchsian differential operators of order $N$,

$$
\mathcal{D}=\partial^{N}+\sum_{i=1}^{N} h_{i}^{\mathcal{D}}(u) \partial^{N-i},
$$

where $\partial=d / d u$, with the following properties.

(a) The singular points of $\mathcal{D}$ are at $b_{0}, \ldots, b_{k}$ only.

(b) The exponents of $\mathcal{D}$ at $b_{s}, s=0, \ldots, k$, are equal to $\mu_{N}^{(s)}, \mu_{N-1}^{(s)}+1$, $\ldots, \mu_{1}^{(s)}+N-1$.

(c) The operator $\mathcal{D}$ is monodromy free.

If $\mathcal{D} \in \Delta_{\mathbf{M}, \boldsymbol{b}}$, then the kernel of $\mathcal{D}$ consists of rational functions with poles at $b_{0}, \ldots, b_{k}$ only.

For a sequence $\boldsymbol{\mu}=\left(\mu_{1}, \ldots, \mu_{N}\right)$ of nonincreasing integers, let $L_{\boldsymbol{\mu}}$ be the irreducible finite-dimensional $\mathfrak{g l}_{N}$-module of highest weight $\boldsymbol{\mu}$. Given a $\mathfrak{g l}_{N}$-module $M$, denote by $M^{\mathfrak{g l}_{N}}$ the subspace of $\mathfrak{g l}_{N}$-invariants in $M$. The subspace $M^{\mathfrak{g l}_{N}}$ is the multiplicity space of the trivial $\mathfrak{g l}_{N}$-module $L_{(0, \ldots, 0)}$ in $M$.

Theorem 6.6. Let $b_{0}, \ldots, b_{k}$ be distinct points on the Riemann sphere which lie on a circle or on a line. Then the set $\Delta_{\mathbf{M}, \boldsymbol{b}}$ consists of $\operatorname{dim}\left(\bigotimes_{s=0}^{k} L_{\boldsymbol{\mu}^{(s)}}\right)^{\mathfrak{g l}_{N}}$ distinct points.

Proof. We reduce the statement to item (i) of Corollary 6.3

Making, if necessary, a fractional-linear change of the variable $u$ in the differential operator $\mathcal{D}$, we can assume without loss of generality that $b_{0}=\infty$ and the points $b_{1}, \ldots, b_{k}$ are on the real axis.

Let $c_{0}, \ldots, c_{k}$ be integers such that $\sum_{s=0}^{k} c_{s}=0$. For $s=0, \ldots, k$, set

$$
\tilde{\boldsymbol{\mu}}^{(s)}=\left(c_{s}+\mu_{1}^{(s)}, \ldots, c_{s}+\mu_{N}^{(s)}\right) .
$$

It is known from the representation theory of $\mathfrak{g l}_{N}$ that the vector spaces

$$
\left(\bigotimes_{s=0}^{k} L_{\boldsymbol{\mu}^{(s)}}\right)^{\mathfrak{g l} \mathfrak{l}_{N}} \text { and }\left(\bigotimes_{s=0}^{k} L_{\tilde{\boldsymbol{\mu}}^{(s)}}\right)^{\mathfrak{g l}_{N}}
$$

are canonically isomorphic. At the same time, we have a bijection $\Delta_{\mathbf{M}, \boldsymbol{b}} \rightarrow \Delta_{\tilde{\mathrm{M}}, \boldsymbol{b}}$ given by the conjugation

$$
\mathcal{D} \mapsto \prod_{s=1}^{k}\left(u-b_{s}\right)^{-c_{s}} \cdot \mathcal{D} \cdot \prod_{s=1}^{k}\left(u-b_{s}\right)^{c_{s}} .
$$


Making transformations (6.3) and (6.4) with $c_{s}=\mu_{N}^{(s)}, s=1, \ldots, k$, we can assume that $\mu_{N}^{(s)}=0, s=1, \ldots, k$. Then $\boldsymbol{\mu}^{(1)}, \ldots, \boldsymbol{\mu}^{(k)}$ are partitions with at most $N$ parts, and the kernel of every operator $\mathcal{D} \in \Delta_{\mathbf{M}, \boldsymbol{b}}$ consists of polynomials only.

If $\mu_{1}^{(0)} \neq 0$, then the set $\Delta_{\mathbf{M}, \boldsymbol{b}}$ is empty and $\operatorname{dim}\left(\bigotimes_{s=0}^{k} L_{\boldsymbol{\mu}^{(s)}}\right)^{\mathfrak{g l}_{N}}=0$. Therefore, the theorem is trivially true.

If $\mu_{1}^{(0)}=0$, then

$$
\boldsymbol{\lambda}=\left(-\mu_{N}^{(0)}, \ldots,-\mu_{1}^{(0)}\right)
$$

is a partition with at most $N$ parts and the space $\left(\bigotimes_{s=0}^{k} L_{\boldsymbol{\mu}^{(s)}}\right)^{\mathfrak{g l}_{N}}$ is canonically isomorphic to the space $\left(\bigotimes_{s=1}^{k} L_{\boldsymbol{\mu}^{(s)}}\right)_{\boldsymbol{\lambda}}^{\text {sing }}$. The set $\Delta_{\mathbf{M}, \boldsymbol{b}}$ coincides with the set $\Delta_{\boldsymbol{\Lambda}, \boldsymbol{\lambda}, \boldsymbol{b}}$ defined in the previous section with $\boldsymbol{\Lambda}=\left(\boldsymbol{\mu}^{(1)}, \ldots, \boldsymbol{\mu}^{(k)}\right)$, and the statement of the theorem follows from item (i) of Corollary 6.3

6.4. Monodromy of eigenspaces of the Bethe algebra. Let $\Theta \subset \mathbb{C}^{n}$ and $\Xi \subset \Omega_{\bar{\lambda}}(\infty)$ be the Zariski open subsets described in Lemma 5.1. Consider the $\operatorname{map} p: \mathbb{C}^{n} \rightarrow \mathbb{C}^{n}, \boldsymbol{b}=\left(b_{1}, \ldots, b_{n}\right) \mapsto \boldsymbol{a}=\left(a_{1}, \ldots, a_{n}\right)$, where $a_{i}$ is the $i$-th elementary symmetric function of $b_{1}, \ldots, b_{n}$. Then $\Pi=p(\Theta)$ is a Zariski open subset of $\mathbb{C}^{n}$.

As vector spaces, $\mathcal{M}_{\boldsymbol{\lambda}, \boldsymbol{a}}$ are identified for all $\boldsymbol{a}$ and $\operatorname{dim} \mathcal{M}_{\boldsymbol{\lambda}, \boldsymbol{a}}=\operatorname{dim}\left(V^{\otimes n}\right)_{\boldsymbol{\lambda}}^{\operatorname{sing}}$. By Lemma 5.1. for any $\boldsymbol{a} \in \Pi$, the vector space $\mathcal{M}_{\boldsymbol{\lambda}, \boldsymbol{a}}$ has a basis consisting of eigenvectors of the Bethe algebra $\mathcal{B}_{\boldsymbol{\lambda}, \boldsymbol{a}}$, and distinct eigenvectors have different eigenvalues. Therefore, all eigenspaces of $\mathcal{B}_{\boldsymbol{\lambda}, \boldsymbol{a}}$ are one-dimensional, and the eigenspaces depend on $\boldsymbol{a}$ holomorphically.

Let $\gamma$ be a loop in $\Pi$ starting at $\boldsymbol{a}$. Analytically continuing along the loop we obtain a permutation of the set of the eigenspaces of $\mathcal{B}_{\boldsymbol{\lambda}, \boldsymbol{a}}$. This construction defines a homomorphism of the fundamental group $\pi_{1}(\Pi, \boldsymbol{a})$ to the symmetric group $S_{\operatorname{dim}} \mathcal{M}_{\boldsymbol{\lambda}, \boldsymbol{a}}$. The image of the homomorphism will be called the monodromy group of eigenspaces of the Bethe algebra.

Proposition 6.7. The monodromy group acts transitively on the set of eigenspaces of the Bethe algebra $\mathcal{B}_{\boldsymbol{\lambda}, \boldsymbol{a}}$.

Proof. Consider the set $\Upsilon$ consisting of all pairs $(\boldsymbol{a}, \ell)$, where $\boldsymbol{a} \in \Pi$ and $\ell$ is an eigenspace of the Bethe algebra acting on $\mathcal{M}_{\boldsymbol{\lambda}, \boldsymbol{a}}$. Define the map $w: \Upsilon \rightarrow$ $\Pi,(\boldsymbol{a}, \ell) \mapsto \boldsymbol{a}$.

By Lemma 5.1, we have a holomorphic bijection $\iota: \Upsilon \rightarrow \Xi$ which sends $(\boldsymbol{a}, \ell)$ to $X$ such that $\mathcal{D}_{\ell}=\mathcal{D}_{X}$. We have $w=\operatorname{Wr}_{\boldsymbol{\lambda}} \circ \iota$, where $\operatorname{Wr}_{\boldsymbol{\lambda}}$ is the Wronski map defined by (3.4).

Let $\left(\boldsymbol{a}, \ell_{1}\right),\left(\boldsymbol{a}, \ell_{2}\right)$ be two eigenspaces of the Bethe algebra acting on $\mathcal{M}_{\boldsymbol{\lambda}, \boldsymbol{a}}$. The subset $\Xi=\mathrm{Wr}_{\boldsymbol{\lambda}}^{-1}(\Pi)$ is a Zariski open subset of an affine cell $\Omega_{\overline{\boldsymbol{\lambda}}}$. In particular, it is path connected. Connect the points $i\left(\boldsymbol{a}, \ell_{1}\right), i\left(\boldsymbol{a}, \ell_{2}\right)$ by a curve $\delta$ in $\Xi$. Then $\gamma=\operatorname{Wr}_{\boldsymbol{\lambda}}(\delta)$ is a loop in $\Pi$ whose monodromy sends $\left(\boldsymbol{a}, \ell_{1}\right)$ to $\left(\boldsymbol{a}, \ell_{2}\right)$.

Consider two Bethe lines $(\boldsymbol{a}, \ell),\left(\boldsymbol{a}, \ell_{2}\right)$ in $\mathcal{M}_{\boldsymbol{\lambda}, \boldsymbol{a}}$, the differential operators $\mathcal{D}_{\ell_{1}}$, $\mathcal{D}_{\ell_{2}}$ associated with the lines, and the loop $\gamma$ constructed in the proof of Lemma 6.7. The differential operators holomorphically depend on $\boldsymbol{a}$.

Corollary 6.8. The analytic continuation of $\mathcal{D}_{\ell_{1}}$ along $\gamma$ equals $\mathcal{D}_{\ell_{2}}$. 


\section{ACKNOWLEDGMENTS}

We are grateful to P. Belkale, L. Borisov, V. Chari, P. Etingof, B. Feigin, A. Kirillov, M. Nazarov and F. Sottile for valuable discussions.

\section{REFERENCES}

[B] P. Belkale, Invariant theory of $\mathrm{GL}(n)$ and intersection theory of Grassmannians, Int. Math. Res. Not. 69 (2004), 3709-3721. MR2099498 (2005h:14117)

[CG] V. Chari, J. Greenstein, Current algebras, highest weight categories and quivers, Adv. Math. 216 (2007), no. 2, 811-840. MR2351379 (2009d:17009)

[CL] V.Chari, S. Loktev, Weyl, Fusion and Demazure modules for the current algebra of $s l_{r+1}$, Adv. Math. 207 (2006), no. 2, 928-960. MR 2271991(2008a:17029)

[CP] V. Chari, A. Pressley, Weyl Modules for Classical and Quantum Affine Algebras, Represent. Theory 5 (2001), 191-223 (electronic). MR1850556 (2002g:17027)

[EG] A. Eremenko, A. Gabrielov, Rational functions with real critical points and the B. and M. Shapiro conjecture in real enumerative geometry, Ann. of Math. (2) 155 (2002), no. 1, 105-129. MR 1888795 (2003c:58028)

[EH] D. Eisenbud, J. Harris, Limit Linear Series: Basic Theory, Invent. Math. 85 (1986), 337-371. MR 846932 (87k:14024)

[F] E. Frenkel, Affine algebras, Langlands duality and Bethe ansatz, XIth International Congress of Mathematical Physics (Paris, 1994), Int. Press, Cambridge, MA (1995), 606-642. MR:1370720 (97k:17036)

[Fu] W. Fulton, Intersection Theory, Springer-Verlag, 1984. MR732620 (85k:14004)

[G] M. Gaudin, La fonction d'onde de Bethe, Masson, Paris, 1983. MR693905 (85h:82001)

[GH] Ph. Griffiths, J.Harris, Principles of Algebraic Geometry, Wiley, 1994 . MR.1288523 (95d:14001)

[HP] W. Hodge and D. Pedoe, Methods of Algebraic Geometry, vol. II, Cambridge University Press, Cambridge, 1952. MR0048065 (13:972c)

[HU] R. Howe, T. Umeda, The Capelli identity, the double commutant theorem, and multiplicity-free actions, Math. Ann. 290 (1991), no. 3, 565-619. MR.1116239 (92j:17004)

$[\mathrm{K}] \quad$ R. Kedem, Fusion products of $\mathfrak{s l}_{N}$ symmetric power representations and Kostka polynomials, Quantum theory and symmetries, World Sci. Publ., Hackensack, NJ (2004), 88-93. MR 2170716

[M] I. G. Macdonald, Symmetric functions and Hall Polynomials, Oxford University Press, 1995. MR:1354144 (96h:05207)

[MNO] A. Molev, M. Nazarov, G. Olshanski, Yangians and classical Lie algebras, Russian Math. Surveys 51 (1996), no. 2, 205-282. MR1401535 (97f:17019)

[MTV1] E. Mukhin, V. Tarasov, A. Varchenko, Bethe Eigenvectors of Higher Transfer Matrices, J. Stat. Mech. (2006), no. 8, P08002, 1-44. MR2249767 (2007h:82021)

[MTV2] E. Mukhin, V. Tarasov, A. Varchenko, The B. and M. Shapiro conjecture in real algebraic geometry and the Bethe ansatz, Preprint math.AG/0512299 (2005), 1-18, to appear in Annals of Mathematics.

[MTV3] E. Mukhin, V. Tarasov, A. Varchenko, A generalization of the Capelli identity, Preprint math/0610799 (2006), 1-14, to appear in Manin Festschrift.

[MTV4] E. Mukhin, V. Tarasov, A. Varchenko, Bethe algebra and algebra of functions on the space of differential operators of order two with polynomial solutions, Selecta Mathematica (N.S.), ISSN 1022-1824 (Print) 1420-9020 (Online) DOI 10.1007/s00029-008-0056-x (2007), 1-24.

[MTV5] E. Mukhin, V. Tarasov, A. Varchenko, On reality property of Wronski maps, arXiv:0710.5856 (2007), 1-20, to appear in Confluentes Mathematici.

[MV1] E. Mukhin and A. Varchenko, Critical Points of Master Functions and Flag Varieties, Communications in Contemporary Mathematics 6 (2004), no. 1, 111-163. MR2048778 (2005b:17052)

[MV2] E. Mukhin, A. Varchenko, Norm of a Bethe vector and the Hessian of the master function, Compos. Math. 141 (2005), no. 4, 1012-1028. MR.2148192 (2006d:82022) 
[MV3] E. Mukhin, A. Varchenko, Multiple orthogonal polynomials and a counterexample to the Gaudin Bethe Ansatz Conjecture, Trans. Amer. Math. Soc. 359 (2007), no. 11, 53835418. MR2327035 (2008g:82035)

[S] F. Sottile, Rational curves on Grassmannians: Systems theory, reality, and transversality, Advances in algebraic geometry motivated by physics (Lowell, MA, 2000), Contemp. Math., 276, Amer. Math. Soc., Providence, RI (2001), 9-42. MR1837108 (2002d:14080)

[T] D. Talalaev, Quantization of the Gaudin System, Preprint hep-th/0404153 (2004), 1-19.

[Tm] H. Tamvakis, The connection between representation theory and Schubert calculus, Enseign. Math. (2) 50 (2004), no. 3-4, 267-286. MR2116717 (2006a:20086)

Department of Mathematical Sciences, Indiana University, Purdue University Indianapolis, 402 North Blackford Street, Indianapolis, Indiana 46202-3216

E-mail address: mukhin@math.iupui.edu

St. Petersburg Branch of Steklov Mathematical Institute, Fontanka 27, St. PetersBURG, 191023, Russia

E-mail address: vt@math.iupui.edu

E-mail address: vt@pdmi.ras.ru

Department of Mathematics, University of North Carolina at Chapel Hill, Chapel Hill, North Carolina 27599-3250

E-mail address: anv@email.unc.edu 